\title{
A coupled distinct lattice spring model for rock failure under dynamic loads
}

\author{
Gao-Feng Zhao ${ }^{\mathrm{a}, *}$, Nasser Khalili ${ }^{\mathrm{a}}$, Jiannong Fang ${ }^{\mathrm{c}}$, Jian Zhao ${ }^{\mathrm{b}}$ \\ a School of Civil and Environmental Engineering, The University of New South Wales (UNSW), Sydney, Australia \\ ${ }^{\mathrm{b}}$ Ecole Polytechnique Federale de Lausanne (EPFL), Laboratory of Rock Mechanics, Station 18, CH-1015 Lausanne, Switzerland \\ ${ }^{\mathrm{c}}$ Ecole Polytechnique Federale de Lausanne (EPFL), Laboratory of Engineering and Environmental Geology, Station 18, CH-1015 Lausanne, Switzerland
}

\section{A R T I C L E I N F O}

\section{Article history:}

Received 13 May 2011

Received in revised form 4 November 2011

Accepted 13 December 2011

Available online 14 January 2012

\section{Keywords:}

Lattice spring model

Numerical manifold method

Coupling

Dynamic failure

\begin{abstract}
A B S T R A C T
It is necessary to take into account the micro discretization of natural rock when studying its macroscopic failure behavior. This requirement has resulted in renewed and increased interest in the discrete or framework/lattice numerical modeling techniques. However, to fully construct a numerical model for practical applications using a discrete numerical model is computationally difficult with current computing technologies. Hence, a coupled model has been developed to overcome this limitation by coupling the Distinct Lattice Spring Model (DLSM) and the Numerical Manifold Method (NMM). In the coupled model, the microscopic discrete model of the rock is represented by a system of discrete particles interacting via springs while the macroscopic level model is represented by the NMM. The proposed model bears a structure of three layers corresponding to the DLSM model, the NMM model, and a model for coupling, respectively. The coupling model is based on a newly developed Particle based Manifold Method (PMM) to bridge the DLSM with the NMM. The proposed coupled model can reduce the computational resources needed for the purely discrete particle based model. This study introduces theoretical aspects of the coupled model together with a few examples to demonstrate its correctness and feasibility.
\end{abstract}

() 2011 Elsevier Ltd. All rights reserved.

\section{Introduction}

Various mechanical phenomena, such as stress wave propagation, crack coalescence, crack bifurcation and fragmentation, occur in rocks under dynamic loads. Accurately modeling these dynamic phenomena is not possible using existing continuum-based numerical methods like the Finite Element Method (FEM) and the Boundary Element Method (BEM). As such, in recent years, there has been renewed interest in discrete or framework/lattice numerical modeling techniques [1-5]. These models have the following characteristics in common: (i) the materials are discretized into particles that are connected through spring-type forces; (ii) the macro-mechanical response is derived from microscopic interactions between particles; (iii) the material failure at the continuous level is captured naturally from the spring failure at the micro-discontinuous level; and (iv) the complex constitutive relationships and contact mechanisms are readily implemented. Due to the discrete nature of these models, they are suitable for simulating complex fracturing of rocks and solids under dynamic loads. However, these models can only simulate small scale models due to computational restrictions; for real applications, many millions of particles are required for an actual system, which results in computational times and memory demands that surpass the

\footnotetext{
* Corresponding author. Tel.: +612 93855022.

E-mail address: gaofeng.zhao@unsw.edu.au (G.-F. Zhao).
}

capacity of modern personal computers. Of course, if super computers are used with parallel implementation of the discrete numerical models (e.g., [6,7]), these problems can be overcome to some degree. However, coupled modeling provides a more exciting and promising solution, which is not constrained by computing capacity limitations at the arithmetic methodology level.

Given the reasons outlined above, coupled numerical techniques are regarded as one of the directions for future development in computational materials science [8-10]. Coupled methods, e.g. multi-scale methods, usually couple atomistic mechanics or quantum theory and classical continuum mechanics [11-13]. A comprehensive review of such methods is given in $[14,15]$. In recent years, a few studies were conducted using coupled numerical techniques for geomaterials. In these models, the microscopic model is the particle based discrete model and the macroscopic continuum model utilized is typically the Finite Element Method (FEM) e.g. [16-20]. The coupling of the Discrete Element Method (DEM) and the FEM emerged in the late 1980s and several different models were proposed, e.g. those in [21-23]. The coupled model used here is defined as a coupled numerical model that links the particle based discrete method (microscopic model) with a macroscopic continuum method. There are three main components in coupled models for geomechanics: a microscopic model, a macroscopic model and an interface/contact/ coupling method. The most widely used technique is to link a particle based discrete model with the FEM by using the contact 
between the FEM block domain and particles around the interface. For example, Onate and Rojek [16] developed a contact algorithm in their model, Yan et al. [17] used the ghost particle method, Lei and Zang [20] used a penalty function method and Elmekati and Shamy [18] and Cai et al. [24] used the contact function provided in commercial codes to undertake a coupled analysis of geotechnical systems. Another recently developed approach is the use of a bridging domain to link two different scale models [19]; this coupling technique is called the bridging domain method, which was first developed by Xiao and Belytschko [25] for coupling Molecular Dynamics (MD) and FEM. Even though there are numerous coupling approaches available, many of them are not complete and further developments are needed. For example, the interface coupling method induces spurious wave. Using the bridging domain method can eliminate the spurious waves, but attention needs to be given to the miss-match between the Degrees Of Freedoms (DOFs) of the FEM and those of the particle DEM. Lagrange multipliers are often used to treat this problem, but at the expense of further complication of the implementation of the coupled model. Moreover, in most coupled models (e.g. [16-20]), the microscopic and macroscopic domains remain unchanged during the computational process and transition from macroscopic level to microscopic model is not considered.

In this paper, a continuum-discontinuum coupled model is developed to simulate rock failure under dynamic loads, to redressing some of the above-mentioned deficiencies. The work is original in the following three ways: Firstly the micro and macro numerical models are based on the newly developed Distinct Lattice Spring Model (DLSM) [5] and the Numerical Manifold Method (NMM) [26] such that the DOFs for each particle in the DLSM are the same as those in the NMM node. This overcome the missmatching problem in [19], and facilitates coupling between micro and macro numerical models. The second novel contribution of this study is the Particle based Manifold Method (PMM), developed to couple the DLSM and NMM. The last contribution is an automatic translation technique devised to transform the PMM model (macro-level model) into the DLSM model during calculations. This paper covers theoretical aspects of the proposed model and gives a few numerical examples to demonstrate the correctness and feasibility of the developed model.

\section{The microscopic and macroscopic models}

\subsection{Basics of elasto-dynamics}

In this section, the basic equations for linear elasto-dynamics are briefly introduced. Consider the elastic body $\Omega$ shown in
Fig. 1. The boundary $\Gamma$ is composed of the traction boundary $\Gamma_{t}$ and the displacement boundary $\Gamma_{u}$. The governing equation of motion, or momentum conservation, for the solid body under the Lagrangian frame of reference is

$\nabla \cdot \boldsymbol{\sigma}+\mathbf{b}=\rho \ddot{\mathbf{u}}$

subject to the boundary condition

$\mathbf{u}=\overline{\mathbf{u}}$ on $\Gamma_{u}$

$\boldsymbol{\sigma} \cdot \mathbf{n}=\overline{\mathbf{t}}$ on $\Gamma_{t}$

where $\nabla$ is the gradient operator with respect to the current position $\mathbf{x}, \mathbf{u}$ is the displacement and $\ddot{\mathbf{u}}$ is the acceleration, $\boldsymbol{\sigma}$ is the Cauchy stress, $\rho$ is the mass density, $\mathbf{b}$ is the body force per unit mass, $\mathbf{n}$ is the outward normal vector on the boundary surface in the current configuration, and $\overline{\mathbf{t}}$ and $\overline{\mathbf{u}}$ are the prescribed tractions and displacements on the corresponding boundaries, respectively.

\subsection{The Distinct Lattice Spring Model (DLSM)}

Here, the DLSM [5] is selected as the microscopic numerical model for the mechanical description of rock under dynamic loads. The dynamic governing Eqs. (1)-(3) are not explicitly solved; rather Newton's second law of motion and the spring-like interaction force between particles is used. This method is briefly introduced in the following sections.

\subsubsection{The physical model}

In the DLSM, the material is discretized into mass particles with different sizes. Whenever the gap between the two particles is smaller than a given threshold value, the two particles are linked together through a bond between their center points (see Fig. 2a), which consists of normal and shear springs. The threshold

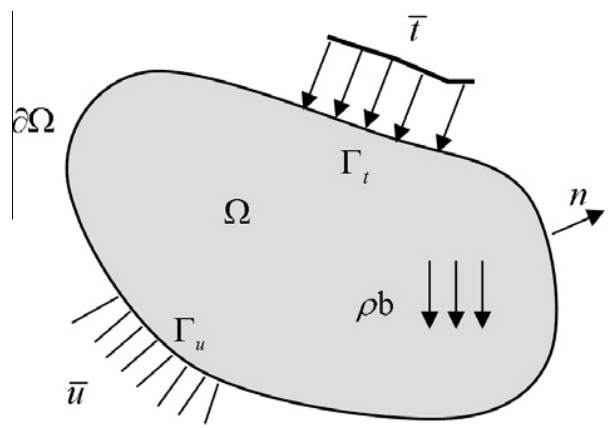

Fig. 1. A solid elastic body under Lagrangian frame.

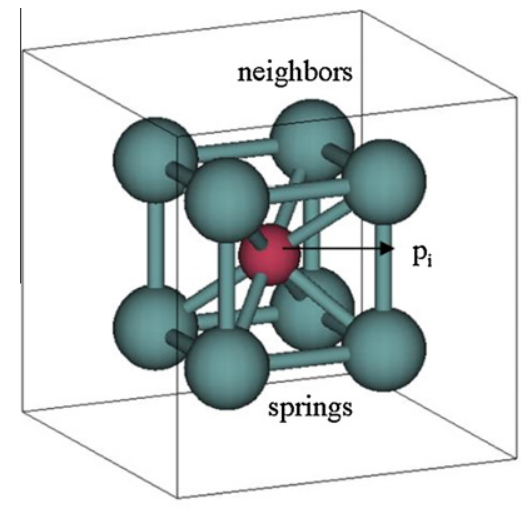

(a) The physical model of DLSM

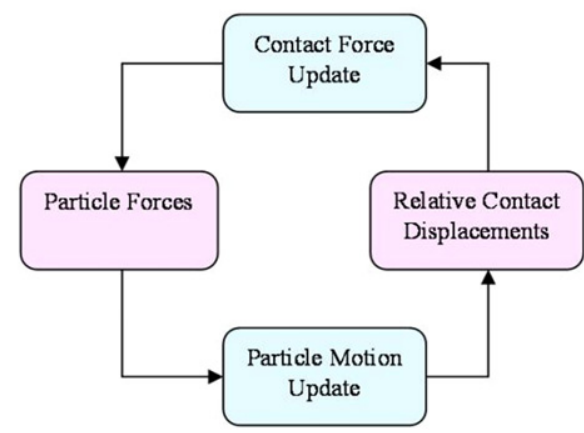

(b) Calculation cycle

Fig. 2. The physical model and the calculation cycle for the DLSM. 


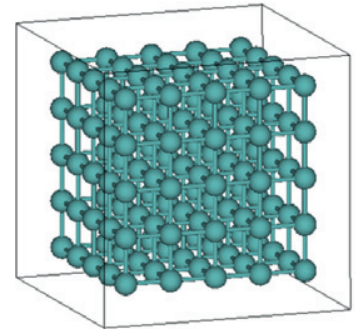

(a) Cubic I

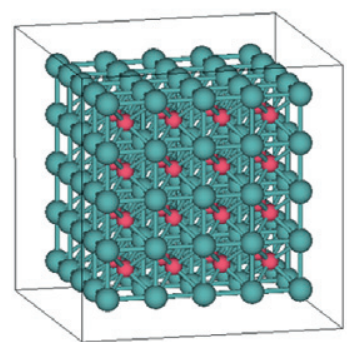

(d) BCC I

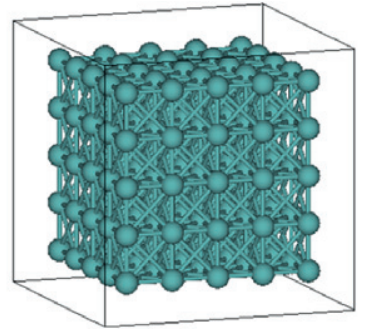

(b) Cubic II

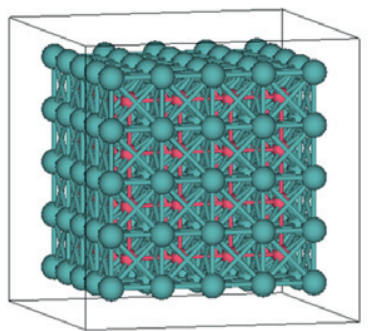

(e) BCC II

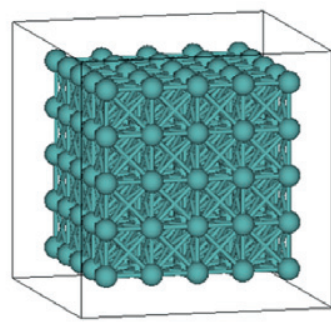

(c) Cubic III

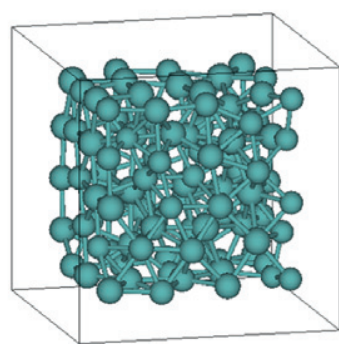

(f) Random structure

Fig. 3. Different lattice structures used in DLSM.

value will influence the lattice structure of the model; different threshold values will produce different lattice structures (see Fig. 3). In the DLSM, the system equation is solved using Newton's second law of motion (the explicit central finite difference scheme). The calculation cycle is illustrated in Fig. 2b. Given the particle displacements (either prescribed initially or obtained from the previous time step), new contacts and broken bonds are detected. The list of neighboring particles for each particle is updated and the contact and spring forces between particles are calculated according to the prescribed force-displacement relations.

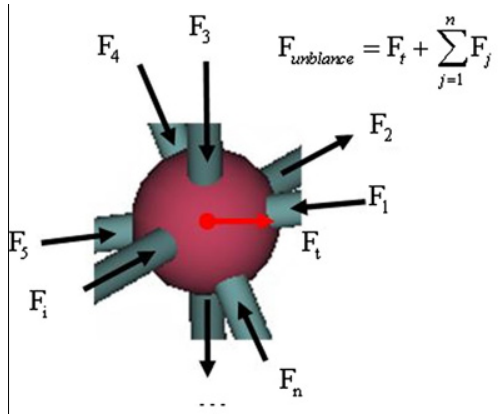

(a) The forces on one particle

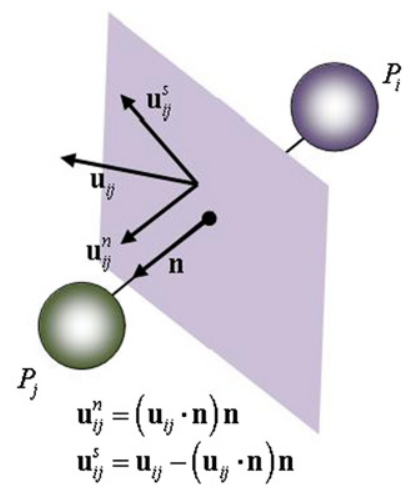

(c) The shear and normal displacement

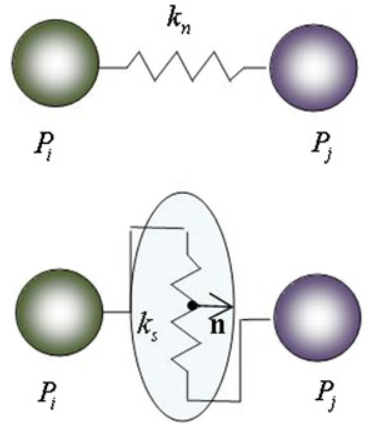

(b) The normal and shear springs
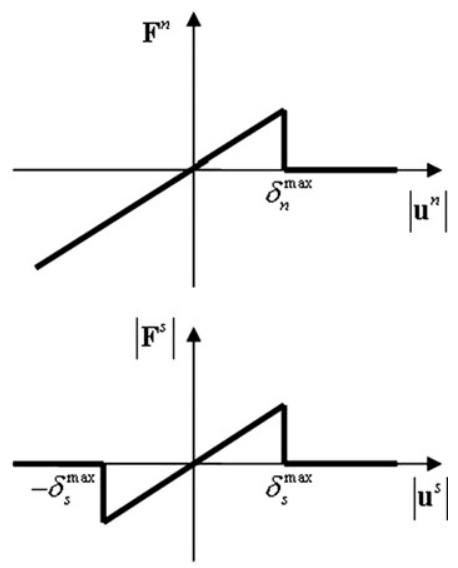

(d) Constitutive laws of lattice springs

Fig. 4. The force and displacement relationships between two particles and the micro constitutive laws. 


\subsubsection{Interactions between particles}

Fig. 4a shows the forces exerted on a single particle. These forces are made up of the external forces and contact forces between particles. The interaction between linked particles is represented by one normal spring and one shear spring as illustrated in Fig. 4b. Unlike the conventional Lattice Spring Models (LSMs), the shear spring is introduced making it possible to handle problems with variable Poisson's ratio. The normal spring is implemented in a conventional way. For a bond connecting particle $i$ and particle $j$, the normal unit vector pointing from particle $i$ to particle $j$ is defined (see Fig. 4c). The relative displacement is calculated as

$\mathbf{u}_{i j}=\mathbf{u}_{j}-\mathbf{u}_{i}$

The normal force between the two particles is defined as

$\mathbf{F}_{i j}^{n}=k_{n} \mathbf{u}_{i j}^{n}$

where $k_{n}$ is the stiffness of the normal spring and $\mathbf{u}_{i j}^{n}=\left(\mathbf{u}_{i j} \bullet \mathbf{n}\right) \mathbf{n}$ is the vector of normal displacement (see Fig. 4c).
For the shear spring, the relative shear displacement between two particles can be obtained simply as $\mathbf{u}_{i j}^{s}=\mathbf{u}_{i j}-\mathbf{u}_{i j}^{n}$ as in some conventional lattice spring models. However, it is easy to show that the shearing force calculated in this way is not rotationally invariant. To overcome this problem, we propose a local strainbased method. Assuming the strain at the two particles is evaluated as $[\boldsymbol{\varepsilon}]_{i}$ and $[\boldsymbol{\varepsilon}]_{j}$ respectively, the strain state of the connecting bond is given as the average of the two particle strains:

$[\boldsymbol{\varepsilon}]_{\text {bond }}=\frac{[\boldsymbol{\varepsilon}]_{i}+[\boldsymbol{\varepsilon}]_{j}}{2 \varepsilon_{x x}}$

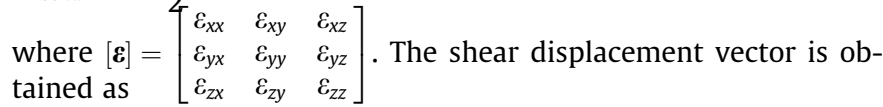
$\hat{\mathbf{u}}_{i j}^{s}=[\boldsymbol{\varepsilon}]_{\text {bond }} \cdot \mathbf{n} l-\left(\left([\boldsymbol{\varepsilon}]_{\text {bond }} \cdot \mathbf{n} l\right) \cdot \mathbf{n}\right) \mathbf{n}$

where $l$ is the initial bond length, i.e. the initial distance between the pair of particles. Thus the shearing force between the two particles is
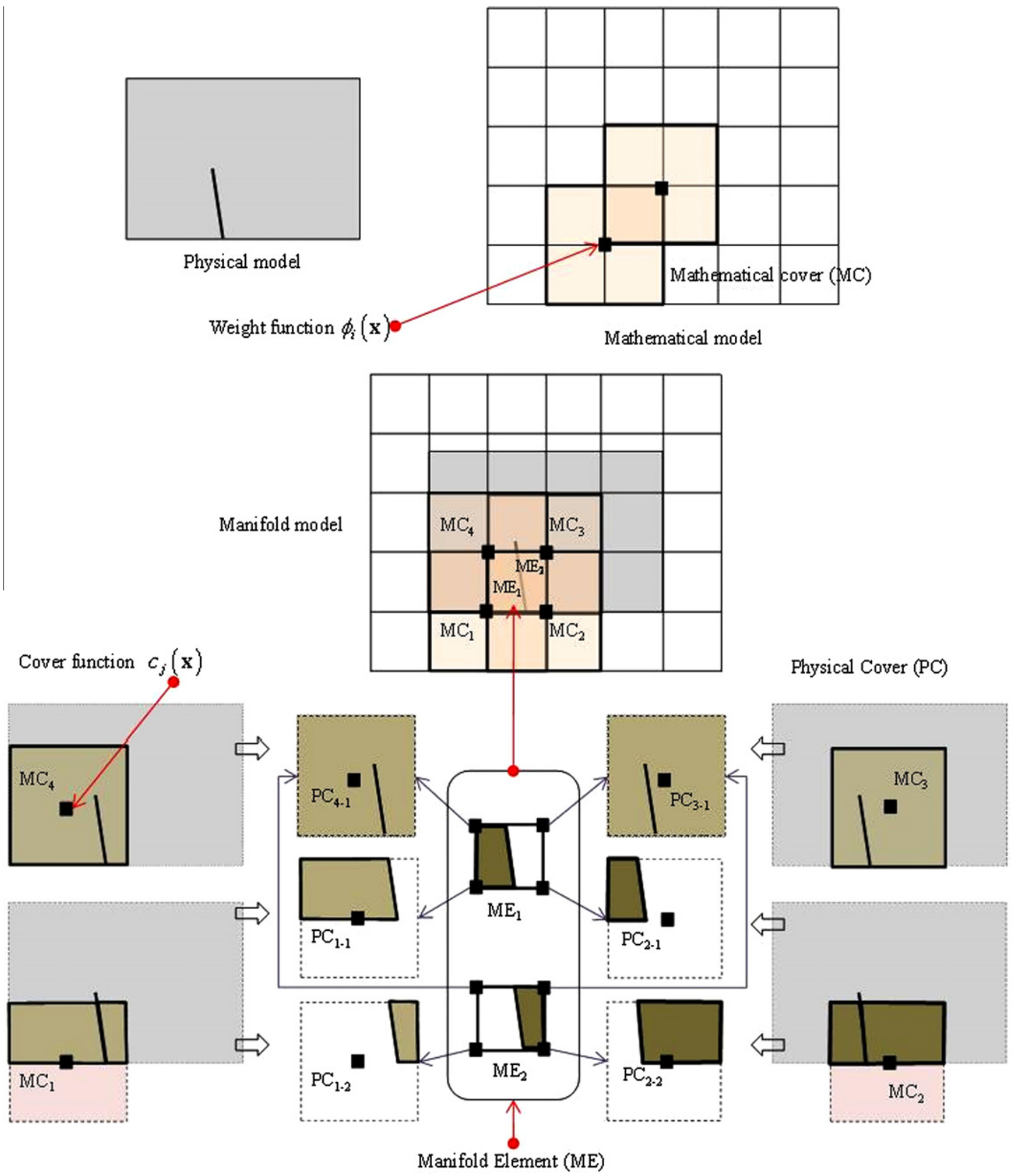

Fig. 5. Components and principle of the NMM. 


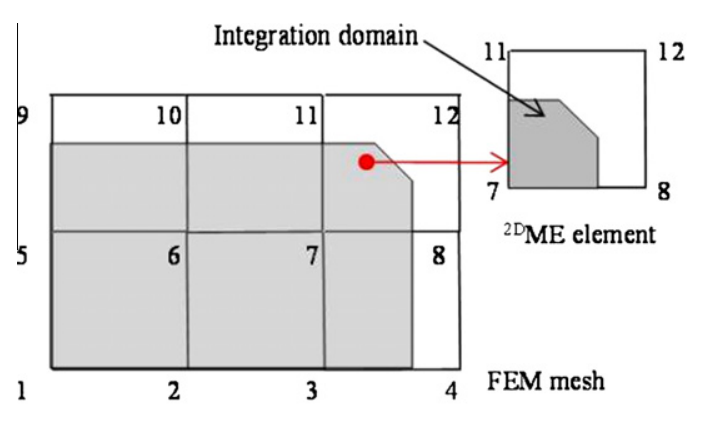

(a) $2 \mathrm{D}$ case

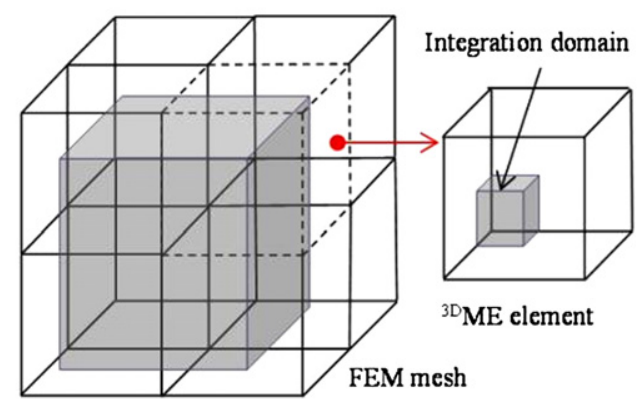

(b) $3 \mathrm{D}$ case

Fig. 6. NMM model based on FEM mesh.

$\mathbf{F}_{i j}^{s}=k_{s} \hat{\mathbf{u}}_{i j}^{s}$

where $k_{s}$ is the stiffness of the shear spring. The proposed method together with the strain calculation procedure using the least square method ensures that the model preserves the rotational invariance. Details on the derivation is presented in Appendix A.

\subsubsection{Damping and time step}

The solution scheme used in the DLSM is conditionally stable. To keep the computation stable, a time step could be chosen according to the requirement that it is less than the time required for elastic wave propagation through the smallest element of the model. This leads to

$\Delta t_{\mathrm{n}}=\min \left(\frac{l_{i}}{C_{p}}\right)$

where $C_{p}$ is the P-wave velocity of the model, $l_{i}$ is the $i$ th spring length of the model. It should be mentioned that the input parameters of the DLSM are macroscopic elastic parameters rather than microscopic spring parameters. This makes the DLSM model consistent with conventional FEM modeling.

Mechanical damping is used in the DLSM to obtain static solutions. For static analysis, the approach is conceptually similar to dynamic relaxation [27]. The equations of motion are damped to reach a force equilibrium state as quickly as possible under the applied initial and boundary conditions. The damping constant (set to 0.8 in the DLSM) is dimensionless and independent of mechanical properties and boundary conditions. The local damping is reported to be under-damped in general.

\subsubsection{Relationship between spring parameters and elastic constants}

In the DLSM, the input elastic parameters are the macro material constants, i.e. the Young's modulus $E$ and the Poisson ratio $v$, in order to keep it consistent with classical FEM. During calculation, the micromechanical parameters are calculated based on the Real Multi-dimensional Bond (RMIB) model [7]. Considering the material heterogeneity, the equations are given as:

$k_{n}=\frac{3}{2 \alpha^{3 D}}\left(\frac{E_{i}}{1-2 v_{i}}+\frac{E_{j}}{1-2 v_{j}}\right)$

$k_{s}=\frac{3}{2 \alpha^{3 D}}\left(\frac{\left(1-4 v_{i}\right) E_{i}}{\left(1+v_{i}\right)\left(1-2 v_{i}\right)}+\frac{\left(1-4 v_{j}\right) E_{j}}{\left(1+v_{j}\right)\left(1-2 v_{j}\right)}\right)$

where $E_{i}$ and $E_{j}$ are the Young's modulus assigned to the linked particles, and $v_{i}$ and $v_{j}$ are the corresponding Poisson's ratios. The $\alpha^{3 D}$ is the microstructure geometry coefficient of the lattice model.

It should be noted that the particle used in DLSM is rigid, and the local strain refers to the local strain of the particle cluster which includes the particle itself and other particles that have intact bonds with the particle. The local strain of one particle is eval- uated by a least square scheme which uses the displacement of the particle cluster. By doing so, discontinuities (e.g. fracture/crack) could be directly considered without using the "visibility criterion" adopted by most meshless methods. As the least squares scheme is used in DLSM, the model can be viewed as a totally meshless method. There is no integration domain and the model only needs a collection of points. In this sense, the DLSM can also be regarded as a new meshless method where the Partial Differential Equations (PDEs) are approximated through a lattice model. The local strain technique allows the DLSM to only use half degree of freedoms compared with the particle DEM (e.g. PFC), and therefore, it is more computationally efficient. Details of DLSM can be found in $[5,7]$.

\subsection{Numerical Manifold Method (NMM)}

The NMM [26] is a numerical method proposed to integrate FEM with the Discontinuous Deformation Analysis (DDA) [28]. It can be regarded as an advanced FEM or Partition of Unity (PU)based FEM. The relationship between PU based FEM and NMM is discussed in detail in Kurumatani and Terada [29]. The basic principle and the components of the approach are shown in Fig. 5. The basic unit in NMM is the manifold element, which is made up of the overlap of the neighboring physical covers. A physical cover is the intersection of the mathematical cover and the physical domain. It is equivalent to the support domain for a node in classical meshless methods. Details of how to construct these manifold elements can also be found in $[29,30]$. The degrees of freedom are defined in these physical covers to represent the deformed state of their physical domains. The meshing methodology is the most distinct feature of NMM, which makes the regular mesh applicable for modeling of an irregular domain. For regular domains with convex

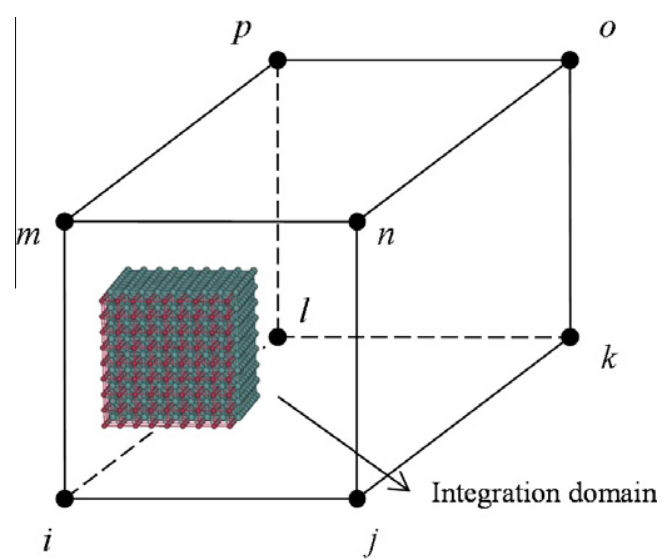

Fig. 7. The PMM element in the coupled DLSM 


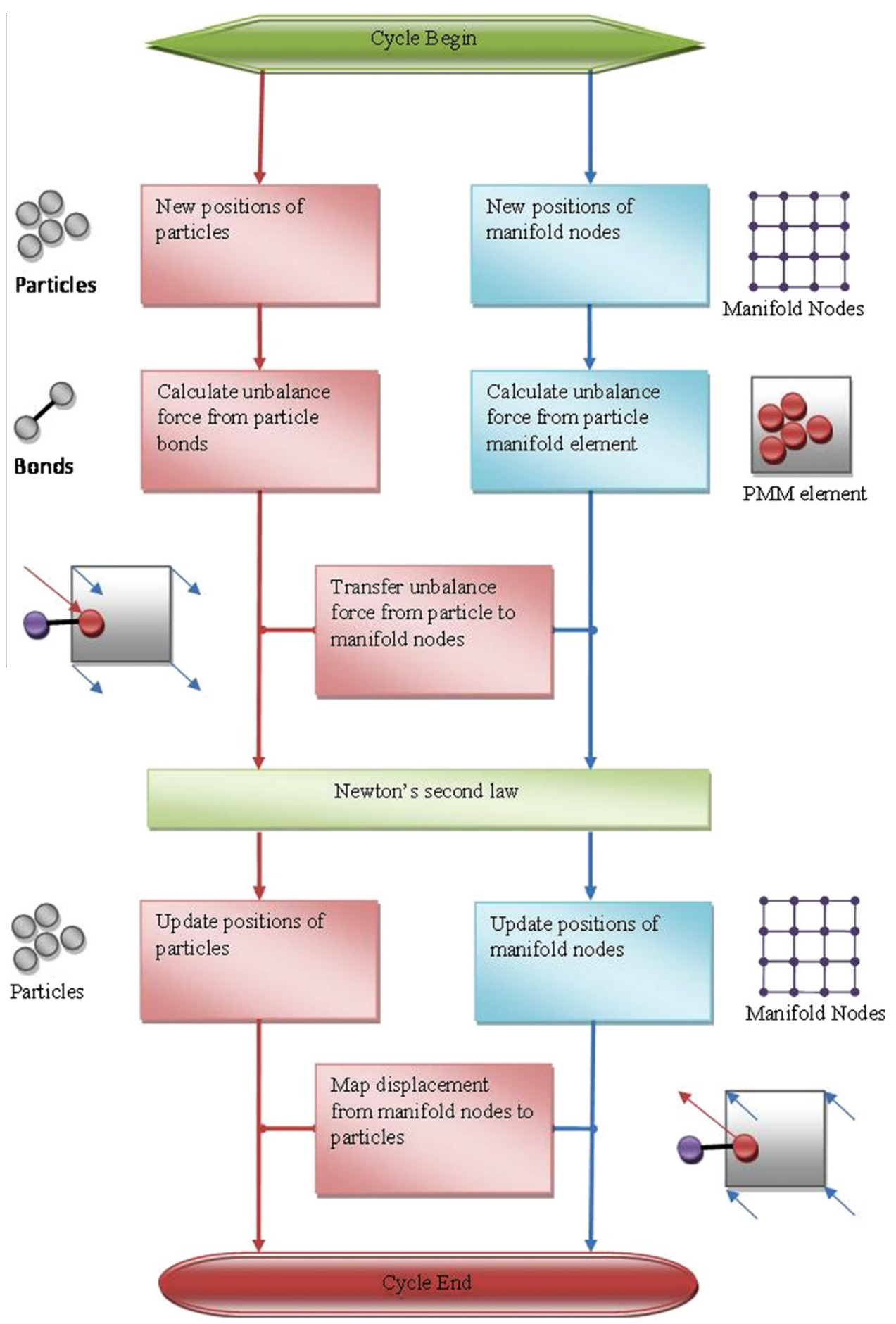

Fig. 8. Coupled calculation cycle in the coupled DLSM.

geometry the application of NMM is identical to that an enriched FEM model (see Fig. 6).

\subsubsection{The explicit NMM}

Traditionally NMM is implemented using an implicit time integration technique suited to modeling large deformation problems. Here, we use an explicit technique to be able to couple the DLSM and NMM seamlessly. In the explicit NMM the approximation function is given in a similar way to the FEM: Firstly, the deformation function is defined in the physical cover (manifold node) as $c_{j}(\mathbf{x})=\sum_{i=1}^{n} b_{j i}(\mathbf{x}) \cdot u_{j i}$

where $c_{j}(\mathbf{x})$ is the displacement function of the $j$ th physical cover, $u_{j i}$ is the general DOFs of the cover, $b_{j i}(\mathbf{x})$ is the basis of the displacement function and $n$ is the number of DOFs. Then, the approximation function of the manifold element can be written as

$u^{h}(\mathbf{x})=\sum_{j=1}^{m} \phi_{j}(\mathbf{x}) c_{j}(\mathbf{x})=\sum_{j=1}^{m} \phi_{j}(\mathbf{x}) \sum_{i=1}^{n} b_{j i}(\mathbf{x}) u_{j i}$ 


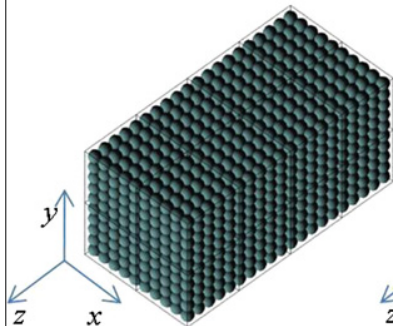

(a) Full DLSM model

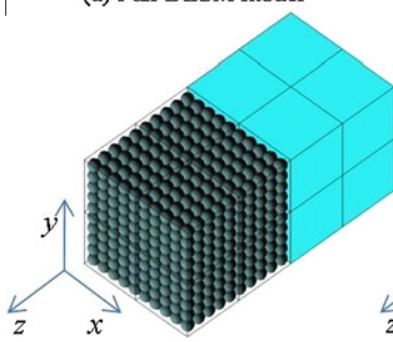

(c) DLSM \& NMM model

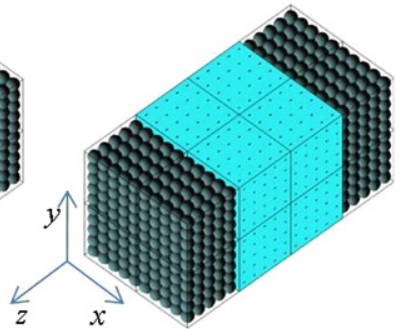

(b) DLSM \& PMM model

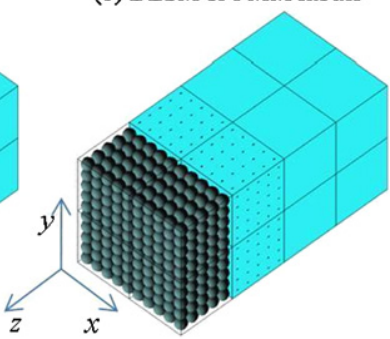

(d) DLSM \& PMM \& NMM model

Fig. 9. Different coupled DLSM models for the bar under tensile loading.
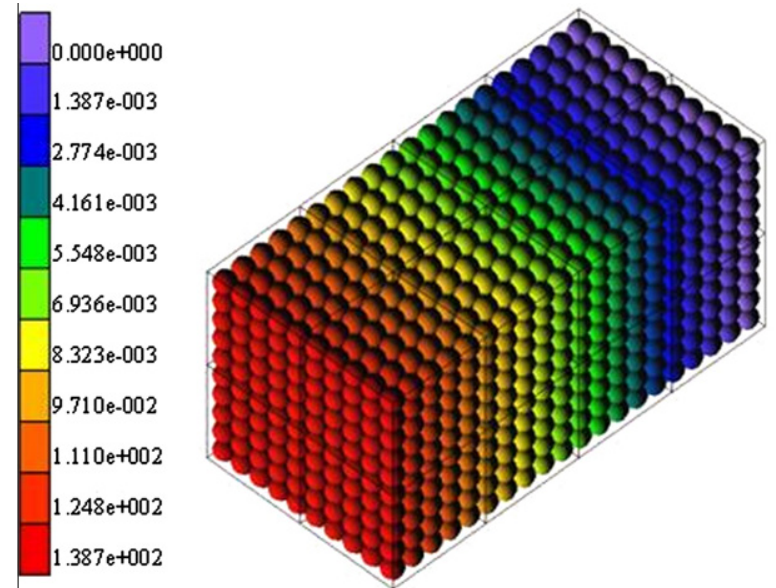

(a) Full DLSM model
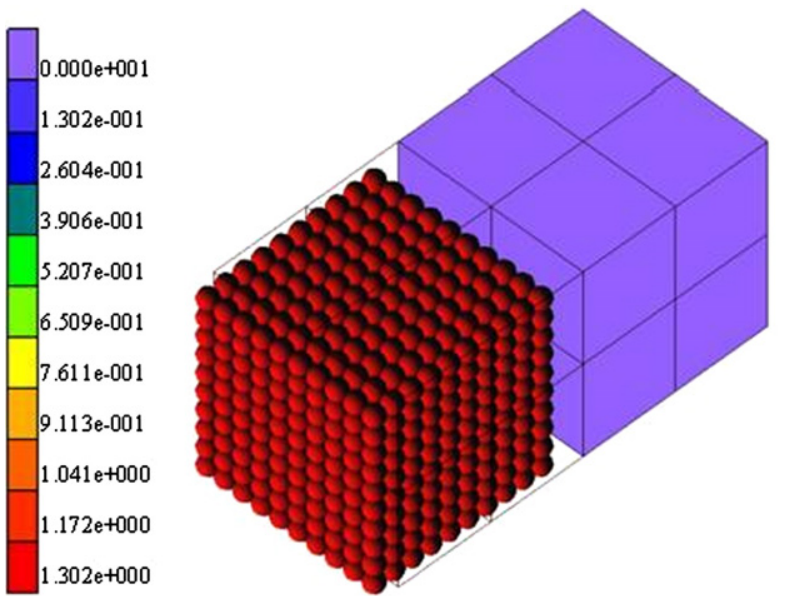

(c) DLSM \& NMM model

where $\phi_{j}$ is the weight function of the cover and $m$ is the number of physical covers of the manifold element. The weight functions must satisfy the partition of unity, namely

$\sum_{j=1}^{m} \phi_{j}(\mathbf{x})=1$

The manifold elements are called three-cover elements or eightcover elements in order to distinguish them from the FEM elements. Equation (13) can then be written in a more familiar form as

$u^{h}(\mathbf{x})=\sum_{k=1}^{n \times m} N_{k}(\mathbf{x}) u_{k}$

where $N_{k}(\mathbf{x})$ is the shape function (the linear order NMM shape functions are exactly the same as the corresponding standard FEM element) and $u_{k}$ is the $k$ th general degree of freedoms of the manifold element. The integration equations can be obtained by applying the weighted residual approach or variation principle and imposing the boundary conditions to Eq. (1). This gives

$$
\begin{aligned}
\int_{\Omega} \rho \mathbf{u}^{*} \cdot \ddot{\mathbf{u}} d V+\int_{\Omega} \nabla \mathbf{u}^{*} & : \boldsymbol{\sigma} d V+\lambda \int_{\Gamma_{u}} \mathbf{u}^{*} \cdot(\mathbf{u}-\overline{\mathbf{u}}) d \Gamma \\
& =\int_{\Omega} \mathbf{u}^{*} \cdot \mathbf{b} d V+\int_{\Gamma_{t}} \mathbf{u}^{*} \cdot \overline{\mathbf{t}} d \Gamma, \forall \mathbf{u}^{*}
\end{aligned}
$$
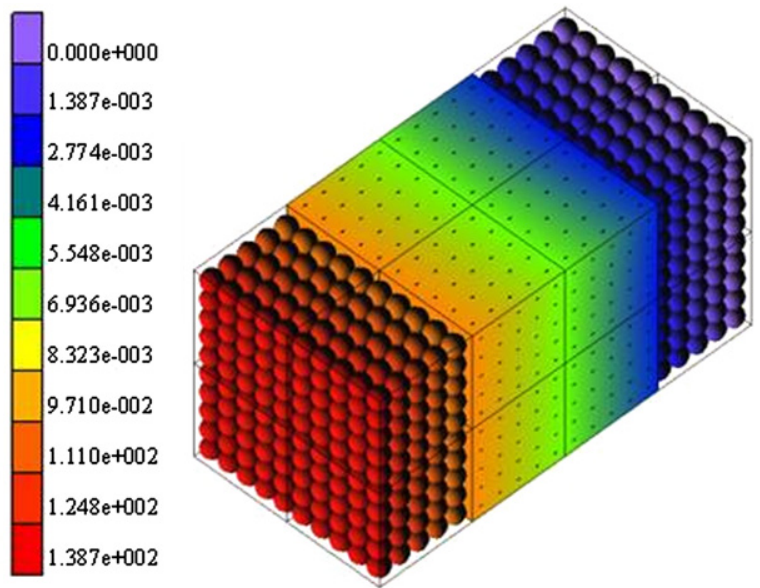

(b) DLSM \& PMM model
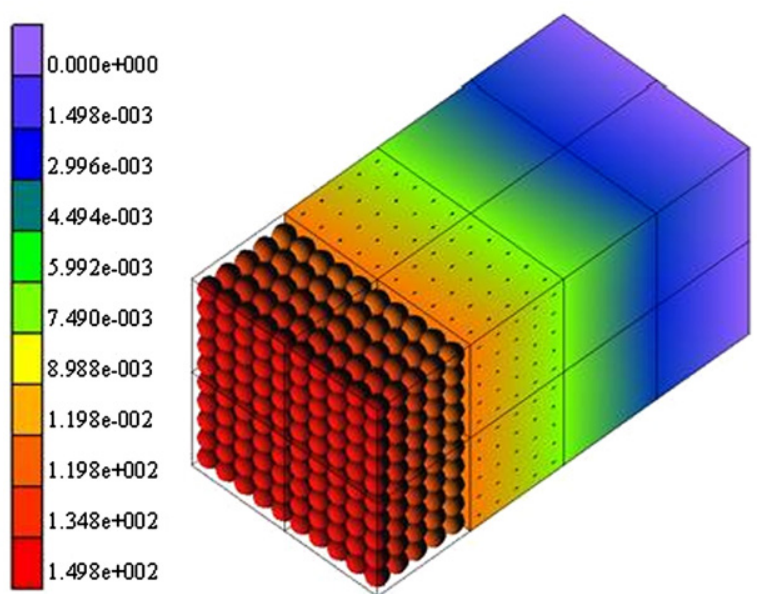

(d) DLSM \& PMM \& NMM model

Fig. 10. Contour maps of the displacement in $z$-direction for the different coupled models. 
Table 1

The predicted $z$ direction displacement using the different coupled DLSM models.

\begin{tabular}{lllll}
\hline Full DLSM & $\begin{array}{l}\text { DLSM \& } \\
\text { PMM }\end{array}$ & $\begin{array}{l}\text { DLSM \& } \\
\text { NMM }\end{array}$ & $\begin{array}{l}\text { DLSM \& } \\
\text { PMM \& } \\
\text { NMM }\end{array}$ \\
\hline$L^{*}(\mathrm{~mm})$ & 18.00 & 18.00 & 19.00 & 19.00 \\
Predicted $(\mathrm{mm})$ & $1.36 \mathrm{e}-2$ & $1.36 \mathrm{e}-2$ & 1.32 & $1.47 \mathrm{e}-2$ \\
Excepted $(\mathrm{mm})$ & $1.44 \mathrm{e}-2$ & $1.44 \mathrm{e}-2$ & $1.52 \mathrm{e}-2$ & $\begin{array}{l}1.52 \mathrm{e}-2 \\
\text { Error }(\%)\end{array}$ \\
\hline
\end{tabular}

where $\mathbf{u}$ is the displacement field and $\mathbf{u}^{*}$ is its variation. The third term in the left-hand side is the penalty term involved in the boundary condition (2). The $\lambda$ is a large number known as the penalty parameter, which is taken as

$\lambda=\beta E$

where $E$ is the elastic module, and $\beta$ is a ratio that is suggested to be from 40 to 100. In NMM, the direct boundary condition can be applied when the manifold nodes (physical cover) are placed exactly on the boundaries. In this case, the third term in the left hand side

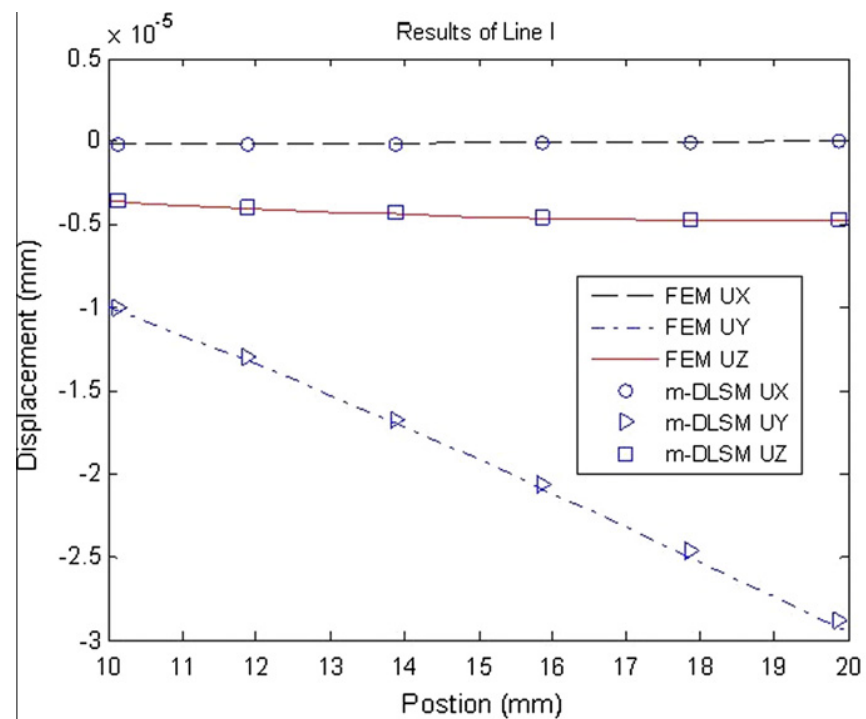

(a) Detection points of Line I

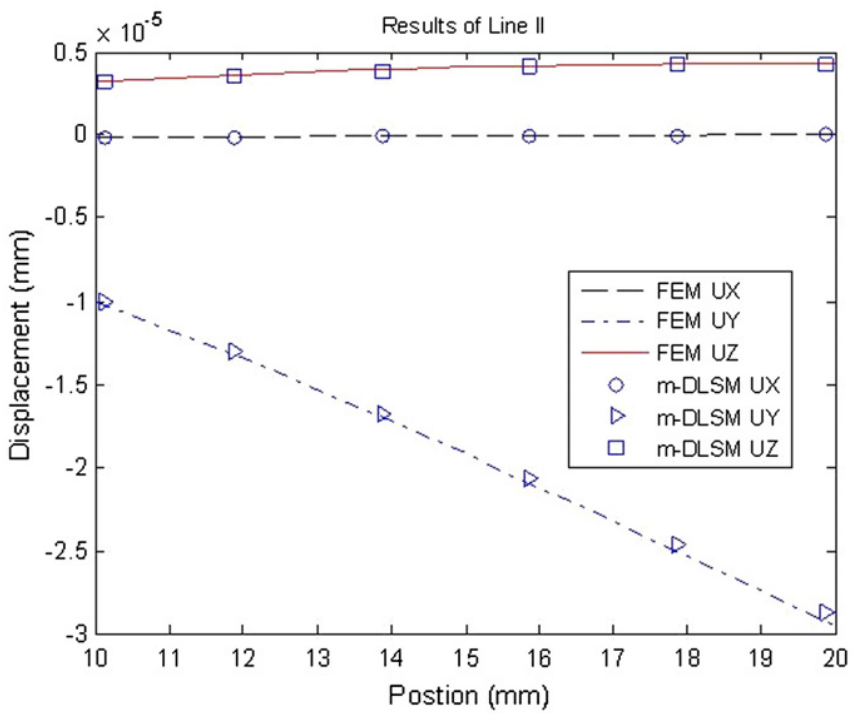

(b) Detection points of Line II

Fig. 11. Full comparison of displacement field predicted by FEM with coupled DLSM for the beam bending problem. of Eq. (16) can be neglected. From Eq. (16), we can now derive the following discretized equation of motion:

$\mathbf{K} \mathbf{u}^{\mathbf{t}}+\mathbf{M} \ddot{\mathbf{u}}^{t}=\mathbf{F}^{t}$

where $\mathbf{u}^{t}$ is the displacement vector and $\ddot{\mathbf{u}}^{t}$ is the acceleration vector. $\mathbf{F}^{t}$ is the external force vector given as

$\mathbf{F}^{t}=\sum_{e=1}^{N_{e l}} \int_{\Omega_{e}} \mathbf{N}_{e}^{T} \mathbf{b} d \Omega_{e}+\sum_{e=1}^{N_{e l}} \int_{\Gamma_{t}} \mathbf{N}_{e}^{T} \overline{\mathbf{t}} d \Gamma_{t}$

The mass matrix $\mathbf{M}$ and stiffness matrix $\mathbf{K}$ are evaluated as follows:

$\mathbf{M}=\sum_{e=1}^{N_{e l}} \rho_{e} \int_{\Omega_{e}} \mathbf{N}_{e}^{T} \mathbf{N}_{e} d \Omega$

$\mathbf{K}=\sum_{e=1}^{N_{e l}} \int_{\Omega_{e}} \mathbf{B}_{e}^{T} \mathbf{D B}_{e} d \Omega$

where $\mathbf{N}_{e}$ and $\mathbf{B}_{e}$ are, respectively, the interpolation matrices of displacement and strain, $\mathbf{D}$ is the elastic matrix and $N_{e l}$ is the number of manifold elements involved in the NMM model.

\subsubsection{Integration and time step}

As stated previously, the NMM can be treated as a FEM where the integration domain of the element is irregular. For this reason, Eqs. (19)-(21) can be integrated through the simplex integration technique or the simplex gauss integration method $[26,28]$. The time integration is achieved using the explicit center difference method as follows:

$\ddot{\mathbf{u}}^{t}=\left(\mathbf{F}^{\mathbf{t}}-\mathbf{K} \mathbf{u}^{t}\right) \mathbf{M}_{\text {lump }}^{-1}$

$\dot{\mathbf{u}}^{t+\Delta t / 2}=\dot{\mathbf{u}}^{t-\Delta t / 2}+\ddot{\mathbf{u}}^{t} \Delta t$

$\mathbf{u}^{t+\Delta t}=\mathbf{u}^{t}+\dot{\mathbf{u}}^{t+\Delta t / 2} \Delta t$

where $\Delta t$ is the time step. The mass matrix is assembled in a lumped form to allow the calculations to be performed element
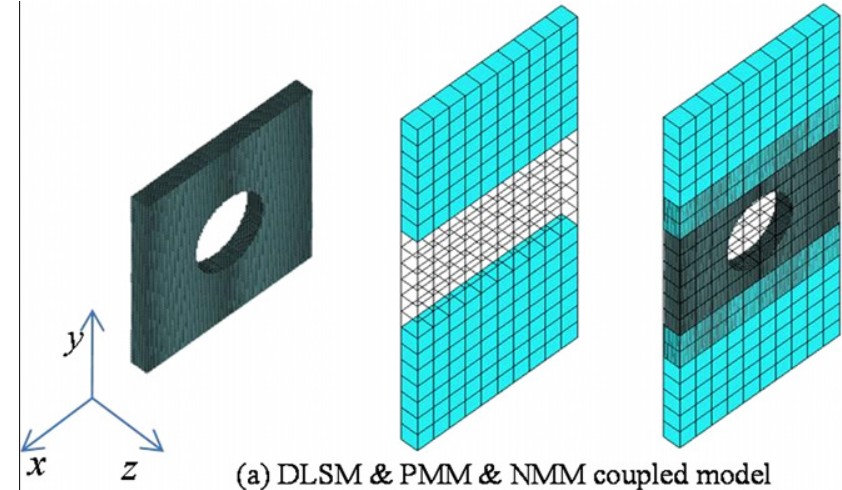

(a) DLSM \& PMM \& NMM coupled model
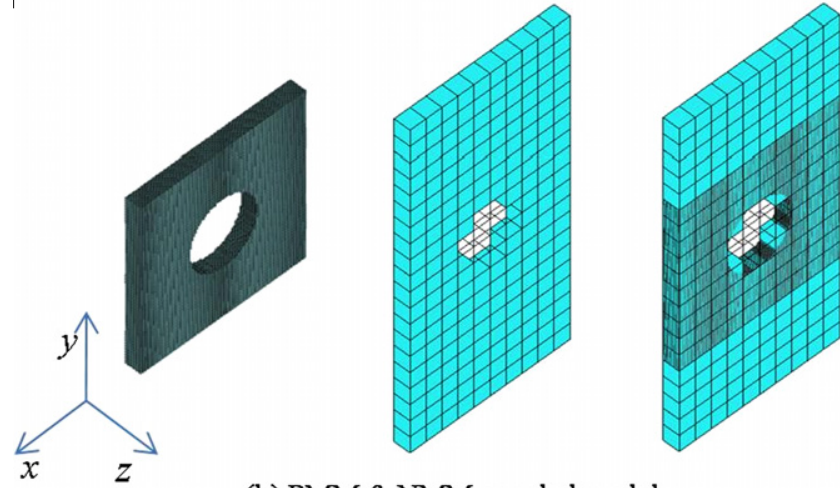

(b) PMM \& NMM coupled model

Fig. 12. Two coupled models for the uniaxial loading of a plate with a circular hole 
by element. The main advantage of the explicit NMM is that the global stiffness matrix does not need to be assembled during the calculations. However, this solution scheme is conditionally stable. As in the DLSM, in order to keep the computation stable, the time step has to be chosen according to following equation:

$\Delta t_{\mathrm{n}} \leqslant \min \left(\frac{l_{i}}{C_{p}}\right)$

where $C_{p}$ is the P-wave velocity of the model and $l_{i}$ is the $i$ th manifold element length of the model.

\subsubsection{Damping}

In order to obtain static solutions in the explicit NMM, a local damping scheme is used to overcome the difficulties of the velocity-proportional damping. The local damping is simply written as

$\sum \mathbf{F}_{i}^{(t)}=\sum \mathbf{F}_{i}^{(t)}-\alpha\left|\sum \mathbf{F}_{i}^{(t)}\right| \operatorname{sgn}\left(\dot{\mathbf{u}}_{i}^{(t-\Delta t / 2)}\right)$ where $\alpha$ is a damping constant which is dimensionless and independent of mechanical properties and boundary conditions. For the dynamic case, the damping term will be switched off $(\alpha=0)$.

\section{The coupled model}

The basic unit in the DLSM is the particle and that of the NMM model is the polyhedral manifold element. Contact detection between these two 3D objects is difficult to implement and is typically handled using complex 3D geometric calculation. On the other hand, direct coupling of these two models can cause a sudden vibration at their interface, leading to generation of spurious solutions. In order to tackle these problems, a method for coupling of the NMM with DLSM is proposed; this method is termed the Particle based Manifold Method (PMM), where the physical domain of the manifold element is replaced by the particle based
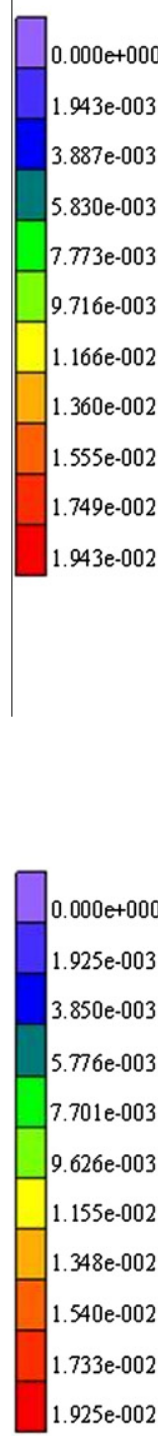
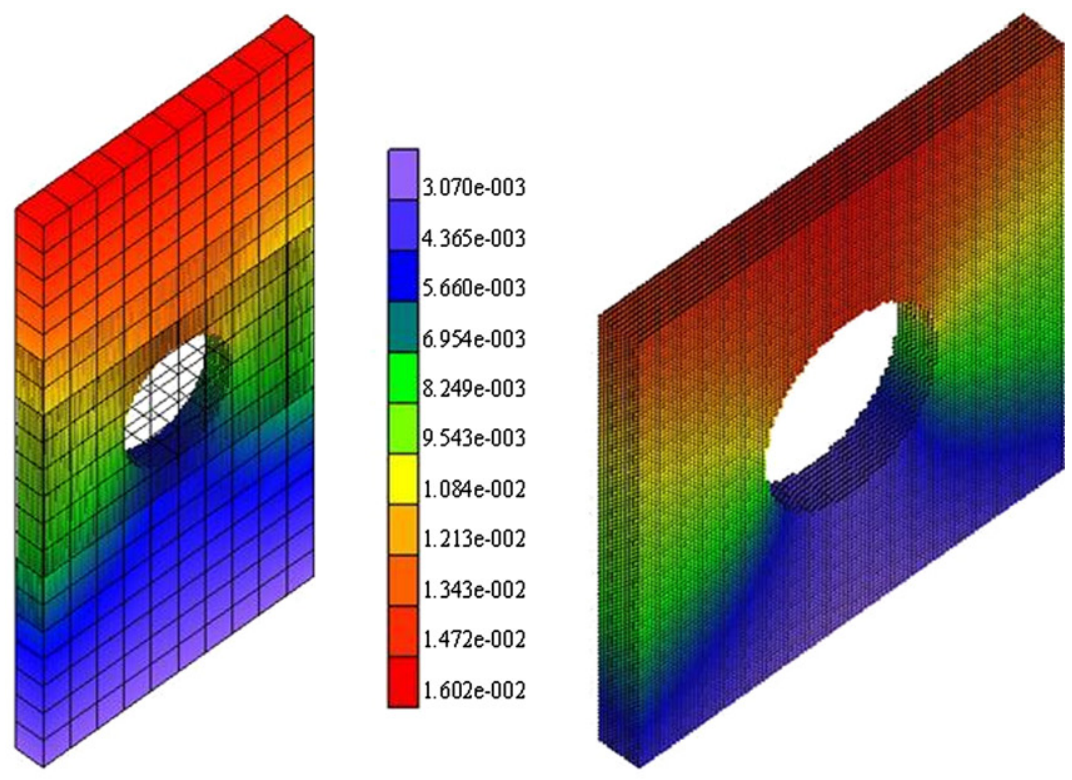

(a) DLSM \& PMM \& NMM coupled model
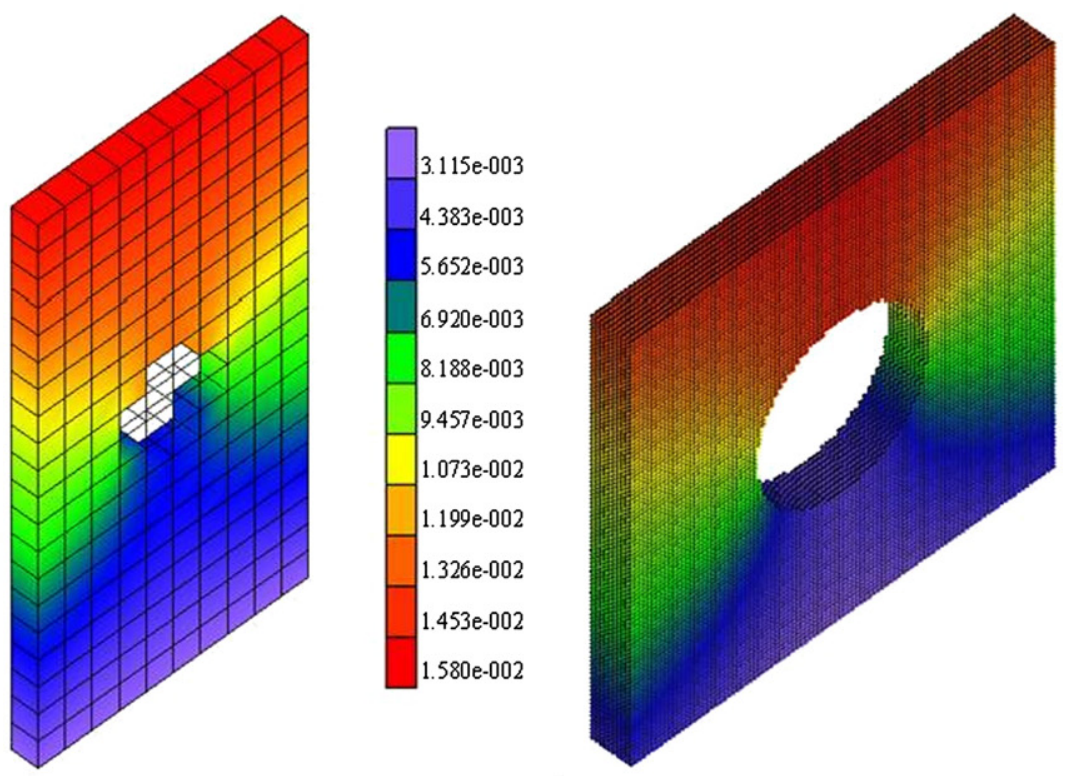

(b) PMM \& NMM coupled model

Fig. 13. The simulation results for the uniaxial loading of a plate with a circular hole problem by the coupled DLSM (contour map of displacement in $y$-direction). 
DLSM model and the DOFs of the model are defined in the physical covers as in the standard NMM. The PMM element is a mixture of the DLSM and manifold elements. By using this element, only sphere-to-sphere contact detection and treatment are required in the coupled model, which is much easier to detect and implement. The PMM model also provides a cushion layer in the coupled model, which naturally bridges the DLSM model and the NMM model. In the following section, the PMM element, the coupled solution procedure and the automatic releasing technique developed for the coupled Distinct Lattice Spring Model (coupled DLSM) will be presented.

\subsection{Particle based Manifold Method (PMM)}

First, the basic idea for the PMM will be introduced. The PMM model is realized by replacing the physical domain of the manifold element by the particle based DLSM model (see Fig. 7). The 3D PMM element used in coupled DLSM is illustrated in Fig. 7. The eight-node FEM element is used as the mathematical element and DLSM model is used as the physical domain.

Since the explicit integration method and lumped mass matrix are used in m-DLSM, the mass matrix of the PMM element is taken as $1 / 8$ of the DLSM model included in the element:

$\mathbf{M}_{i}^{\mathbf{P M E}}=\operatorname{diag}\left(\frac{1}{8} \sum_{j=1}^{m_{i}} m_{i j}^{p}\right)$

where $\mathbf{M}_{i}^{\mathbf{P M E}}$ is the mass matrix of the PMM element, $m_{i}$ is the number of particles included in the PMM element and $m_{i j}^{p}$ is the mass of the particle. The stiffness matrix of the PMM element has to be obtained in a distinct way as the deformation energy of the DLSM model is stored on the network of bonds between particles. The integration domain of the PMM element is neither 2D nor 3D; due to the discrete natural property of the lattice network, the integration is realized through a summation operation as

$\mathbf{K}_{i}^{\mathrm{PME}}=\sum_{j=1}^{n_{i}} \mathbf{K}_{i j}^{\mathrm{b}}$

where $\mathbf{K}_{i}^{\mathrm{PME}}$ is the stiffness matrix of the PMM element, $n_{i}$ is the number of bonds included in the PMM element and $\mathbf{K}_{i j}^{\mathbf{b}}$ is the stiffness matrix contributed by each lattice bond (a pair of normal and shear springs).
The stiffness of the bond in local coordinates is

$\mathbf{K}_{i j}^{\text {bond }}=\left(\begin{array}{cccc}k_{n} & 0 & 0 & 0 \\ 0 & k_{s} & 0 & 0 \\ 0 & 0 & k_{s} & 0 \\ 0 & 0 & 0 & k_{s}\end{array}\right)$

and the bond deformation is represented as

$\mathbf{u}^{\text {bond }}=\left(u^{n}, u_{x}^{s}, u_{y}^{s}, u_{z}^{s}\right)$

It should be mentioned that the shear spring in the DLSM model is a vector spring whose deformation is represented by a vector with three variables. The strain state of the PMM element is given as

$\boldsymbol{\varepsilon}=\left(\begin{array}{llllll}\varepsilon_{x x} & \varepsilon_{y y} & \varepsilon_{z z} & \varepsilon_{x y} & \varepsilon_{y z} & \varepsilon_{x z}\end{array}\right)^{T}=\mathbf{B}^{*} \mathbf{u}^{\mathrm{ME}}$

where $\mathbf{u}^{\mathrm{ME}}$ is the node displacement of the PMM element, $\mathbf{B}^{*}=\left[\mathbf{B}_{i}\right]$ is the strain interpolation matrix of the mathematical element, which can be obtained as

$\mathbf{B}_{i}=\left(\begin{array}{ccc}N_{i, x} & 0 & 0 \\ 0 & N_{i, y} & 0 \\ 0 & 0 & N_{i, z} \\ \frac{1}{2} N_{i, y} & \frac{1}{2} N_{i, x} & 0 \\ 0 & \frac{1}{2} N_{i, z} & \frac{1}{2} N_{i, y} \\ \frac{1}{2} N_{i, z} & 0 & \frac{1}{2} N_{i, x}\end{array}\right)$

where $N_{i, x}, N_{i, y}$ and $N_{i, z}$ are derivatives of the shape functions. The shape functions $N_{i}$ and their corresponding derivatives are provided in Appendix B.

The bond deformation vector can then be represented by

$\mathbf{u}^{\text {bond }}=\mathbf{L}_{4 \times 6} \boldsymbol{\varepsilon}$

where $\mathbf{L}_{4 \times 6}$ is a transformation matrix. Based on Eq. (7), $\mathbf{L}_{4 \times 6}$ is obtained as

$$
\begin{aligned}
\mathbf{L}_{4 \times 6} & =\mathbf{T}_{4 \times 3} \mathbf{Q}_{3 \times 6}=l_{i j}\left(\begin{array}{ccc}
n_{x} & n_{y} & n_{z} \\
1-n_{x}^{2} & -n_{x} n_{y} & -n_{x} n_{z} \\
-n_{y} n_{x} & 1-n_{y}^{2} & -n_{y} n_{z} \\
-n_{z} n_{x} & n_{z} n_{y} & 1-n_{z}^{2}
\end{array}\right) \\
& \times\left(\begin{array}{cccccc}
n_{x} & 0 & 0 & n_{y} & 0 & n_{z} \\
0 & n_{y} & 0 & n_{x} & n_{z} & 0 \\
0 & 0 & n_{z} & 0 & n_{y} & n_{x}
\end{array}\right)
\end{aligned}
$$

Table 2

\begin{tabular}{|c|c|c|c|c|c|c|}
\hline & Point A & Point B & Point C & Point D & Point E & Point F \\
\hline DLSM \& PMM \& NMM (mm) & $9.58 \mathrm{e}-4$ & $9.60 e-4$ & $9.62 \mathrm{e}-4$ & $9.60 e-4$ & $9.60 \mathrm{e}-4$ & $9.58 \mathrm{e}-4$ \\
\hline PMM \& NMM (mm) & $9.50 e-4$ & $9.52 e-4$ & $9.53 e-4$ & $9.53 e-4$ & $9.52 \mathrm{e}-4$ & $9.50 \mathrm{e}-4$ \\
\hline Percentage difference (\%) & 0.84 & 0.83 & 0.94 & 0.73 & 0.83 & 0.84 \\
\hline
\end{tabular}

Displacements in the $y$-direction of the plane predicted by different models.

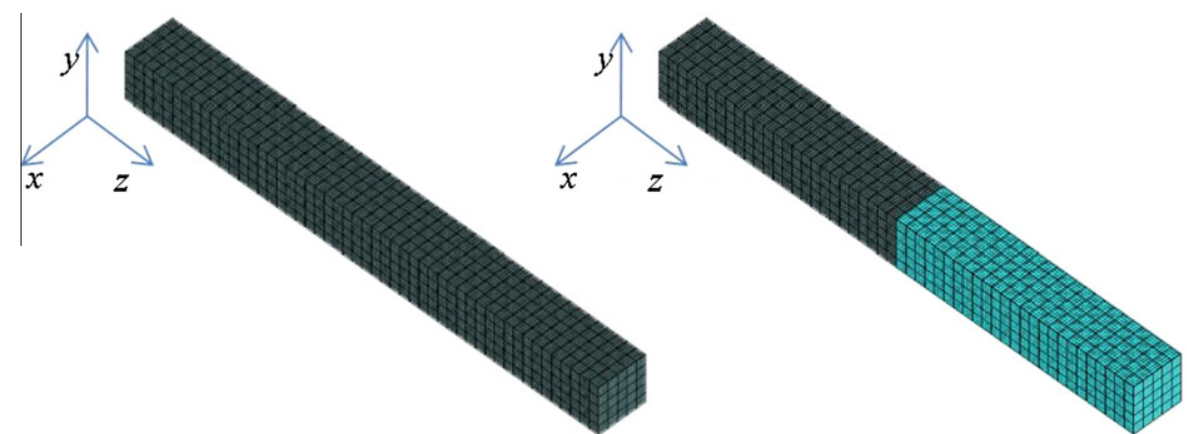

(a) Full DLSM model (b) DLSM \& PMM model

Fig. 14. The coupled DLSM models for wave propagation through an elastic bar. 

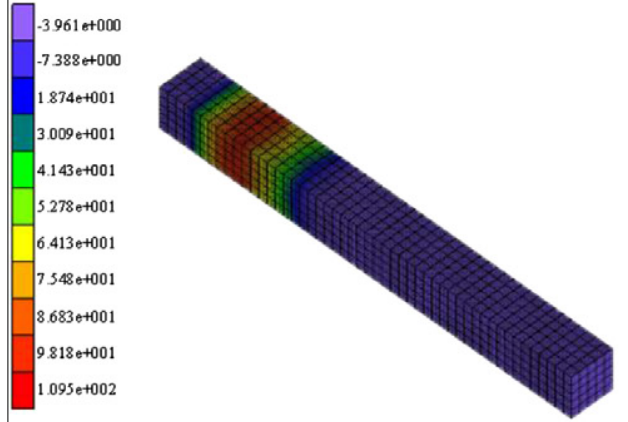

$\mathrm{t}=5 \mu \mathrm{s}$

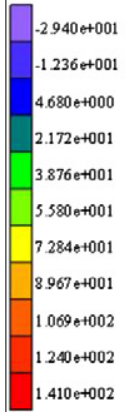

$10 \mathrm{e}+002$

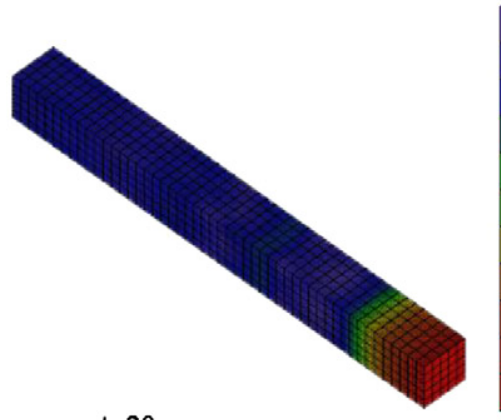

$\mathrm{t}=20 \mu \mathrm{s}$

(a) Full DLSM model
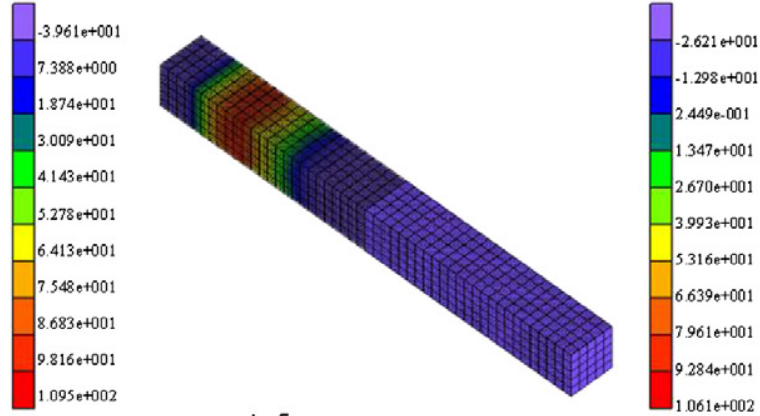

$\mathrm{t}=5 \mu \mathrm{s}$
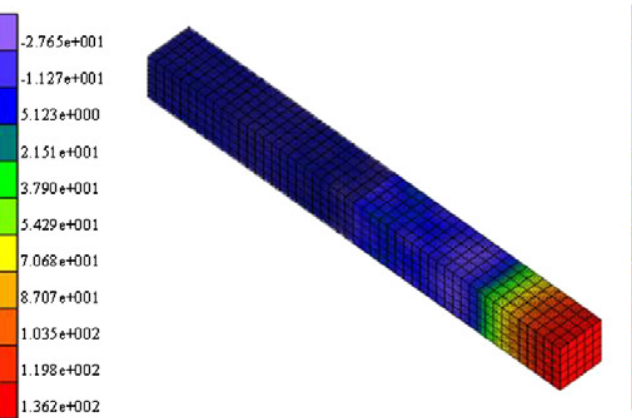

$\mathrm{t}=20 \mu \mathrm{s}$
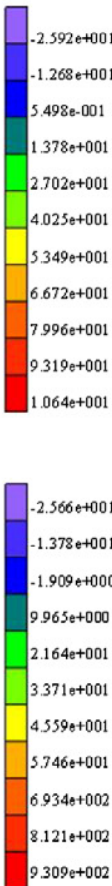

$9.309+002$

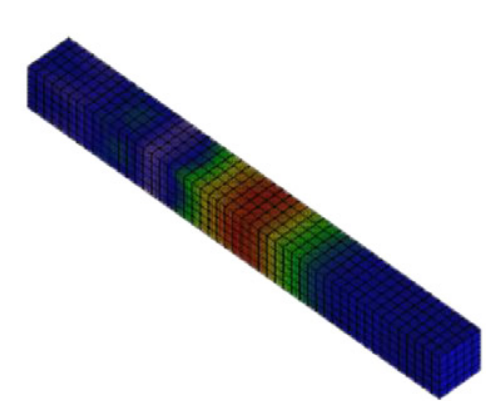

$t=12 \mu \mathrm{s}$

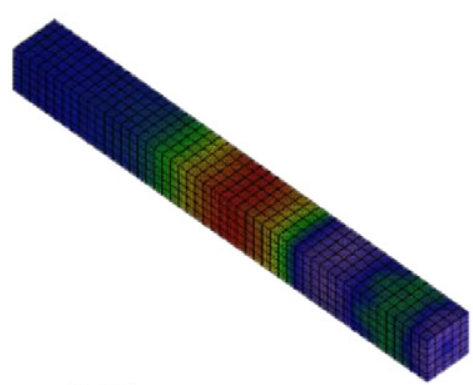

$\mathrm{t}=30 \mu \mathrm{s}$

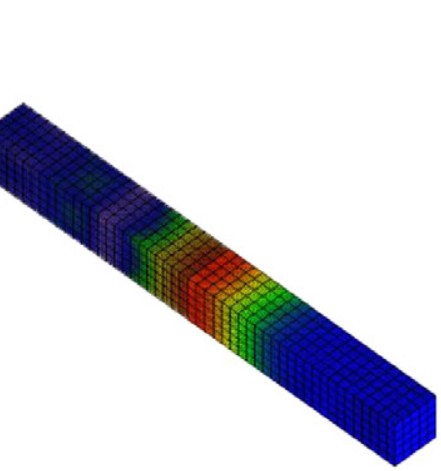

$\mathrm{t}=12 \mu \mathrm{s}$

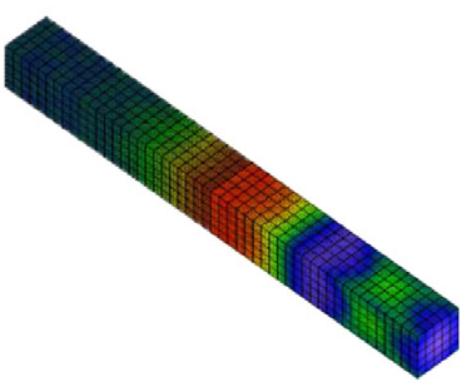

$\mathrm{t}=\mathbf{3 0 \mu \mathrm { s }}$

(b) DLSM \& PMM model

Fig. 15. Wave propagation through an elastic bar simulated using coupled DLSM (contour map of velocity in $z$ direction).

where $l_{i j}$ is the length of the bond and $\left(n_{x}, n_{y}, n_{z}\right)$ is the normal vector of the bond, it is defined as

$\mathbf{n}=\left(n_{x}, n_{y}, n_{z}\right)=\left(\frac{x_{2}-x_{1}}{l}, \frac{y_{2}-y_{1}}{l}, \frac{z_{2}-z_{1}}{l}\right)$

where $\left(x_{1}, y_{1}, z_{1}\right)$ and $\left(x_{2}, y_{2}, z_{2}\right)$ are the coordinates for two particles.
Now, the strain energy of the bond can be written as

$$
\Pi_{\mathrm{b}}=\frac{1}{2}\left(\mathbf{K}_{i j}^{\text {bond }}\left(\mathbf{L B}^{*} \mathbf{u}^{\mathrm{ME}}\right)\right)^{T}\left(\mathbf{L B}^{*} \mathbf{u}^{\mathrm{ME}}\right)
$$

Finally, the contribution of each bond to the stiffness matrix is obtained from the energy minimization principle as 


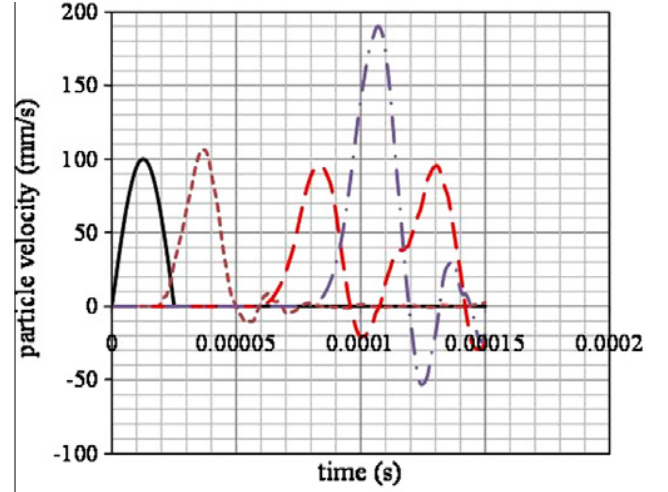

(a) Full DLSM model

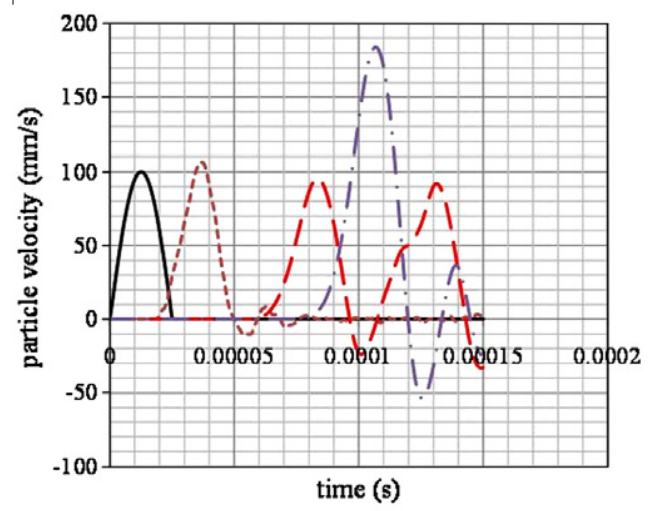

(b) DLSM \& PMM model

Fig. 16. Simulation results of the wave propagation using the coupled DLSM.

$\mathbf{K}_{i j}^{\mathrm{b}}=\left[\frac{\partial^{2} \Pi_{\mathrm{b}}}{\partial u_{i} \partial u_{j}}\right]=\left(\mathbf{L B}^{*}\right)^{T} \mathbf{K}_{i j}^{\mathrm{bond}} \mathbf{L B}^{*}$

\subsection{Scheme of the coupling procedure}

Fig. 8 shows the work flow of the coupled calculation cycle in the m-DLSM. The DLSM and NMM computations are performed in parallel and interactions between the two models are captured through the PMM. Information exchange occurs only at the beginning and the end of each cycle. The mapping of unbalanced forces from particles to PMM elements is realized using

$\mathbf{F}_{i}^{\mathrm{ME}}=\mathbf{N}_{i j} \mathbf{F}_{i j}^{\mathrm{LS}}$

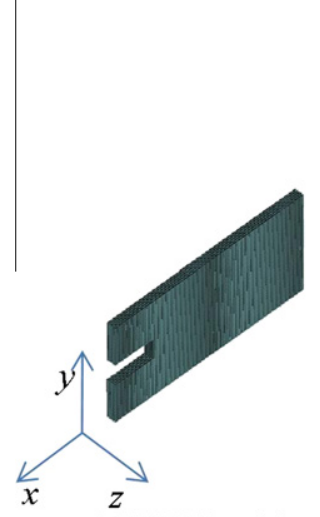

(a) DLSM model

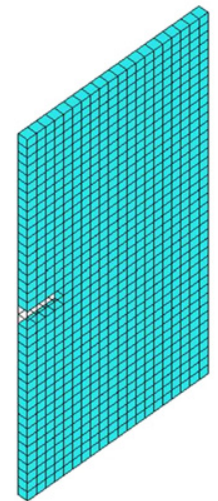

(b) NMM model

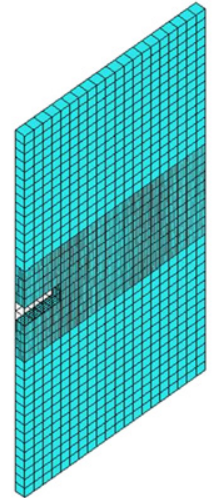

(c) Coupled model
Fig. 17. The coupled model for a solid specimen with a side notch.

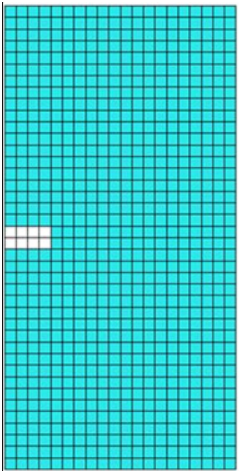

(a) $\operatorname{step}=10$

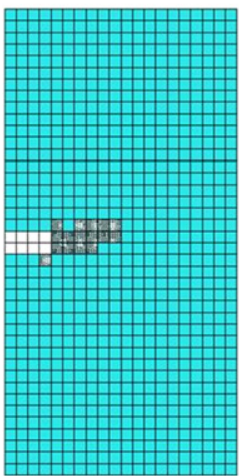

(d) step $=40$

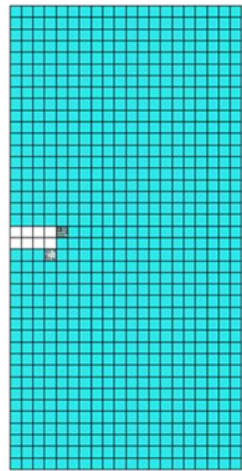

(b) $\mathrm{step}=20$

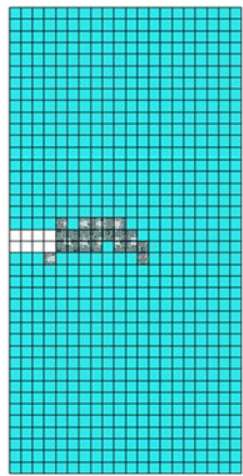

(e) $\operatorname{step}=50$

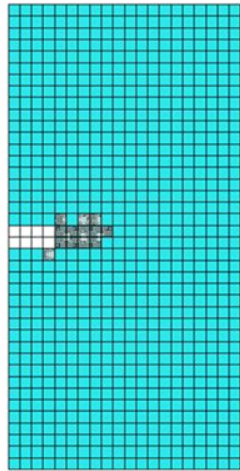

(c) step $=30$

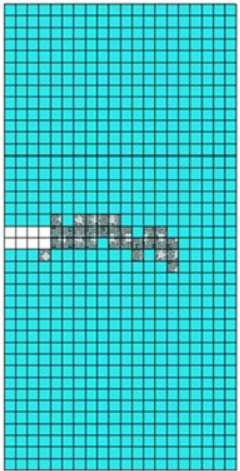

(f) $\operatorname{step}=60$
Fig. 18. PMM releasing process for the coupled DLSM.

where $\mathbf{F}_{i}^{M E}$ (a vector of 24 components) is the force transferred to each node of the $i$ th PMM element, $\mathbf{N}_{i j}$ is an interpolation matrix at the particle $j$ and $\mathbf{F}_{i j}^{\mathrm{LS}}$ is the calculated unbalance force on the particle $j$ due to its bond connections with the DLSM particles. $\mathbf{N}_{i j}$ is given as

$$
\left[\mathbf{N}_{i j}\right]^{T}=\left[\begin{array}{ccccccc}
N_{1}\left(\mathbf{x}_{j}\right) & 0 & 0 & \cdots & N_{8}\left(\mathbf{x}_{j}\right) & 0 & 0 \\
0 & N_{1}\left(\mathbf{x}_{j}\right) & 0 & \cdots & 0 & N_{8}\left(\mathbf{x}_{j}\right) & 0 \\
0 & 0 & N_{1}\left(\mathbf{x}_{j}\right) & \cdots & 0 & 0 & N_{8}\left(\mathbf{x}_{j}\right)
\end{array}\right]
$$

After obtaining the unbalanced forces on the particles and manifold nodes, new positions of these particles and manifold nodes are obtained using Newton's second law. Then, the displacement of the NMM model is mapped to the particles which are located in the PMM model. The mapping operation is given as

$\mathbf{u}_{i j}^{\mathrm{LS}}=\left[\mathbf{N}_{i j}\right]^{T} \mathbf{u}_{i}^{\mathrm{ME}}$

where $\mathbf{u}_{i j}^{\mathrm{LS}}$ is the mapped displacement from the PMM model to the linked particle and $\mathbf{u}_{i}^{\mathrm{ME}}$ is displacement vector of the PMM element. The interaction between the PMM and the DLSM is achieved through the interaction of the DLSM particle with the PMM particle while the interaction between the PMM and the NMM is realized by sharing common manifold nodes. The PMM model is used as the mid-scale layer to attain coupling of the DLSM and the NMM.

The time step is selected as the minimum value of the time step used for the NMM and the DLSM models. In practical applications, the time step of the DLSM model is typically selected since the size of the DLSM particles is always smaller than the NMM element size. 


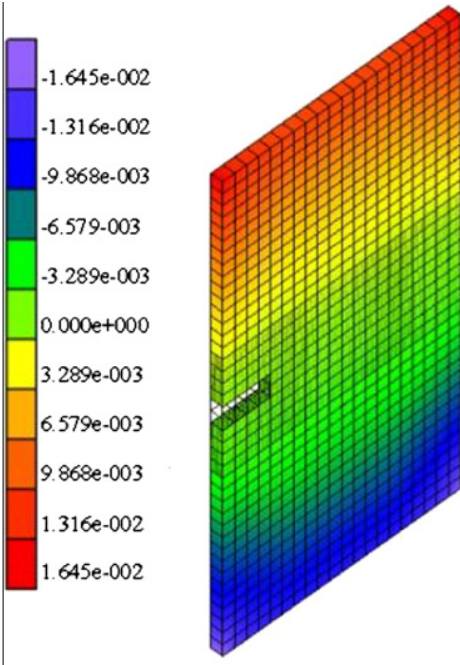

(a) $\operatorname{step}=10$
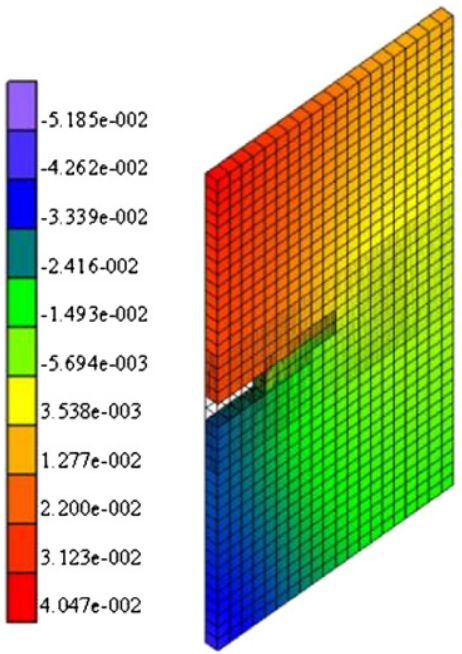

(d) step $=40$

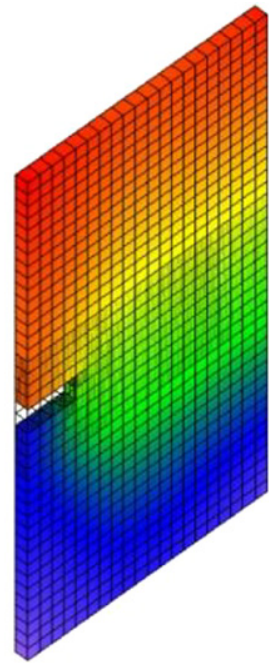

(b) $\operatorname{step}=20$

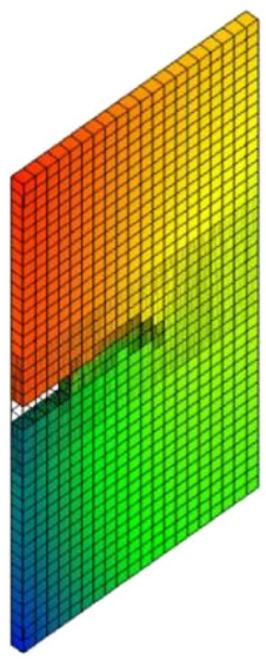

(e) step $=50$

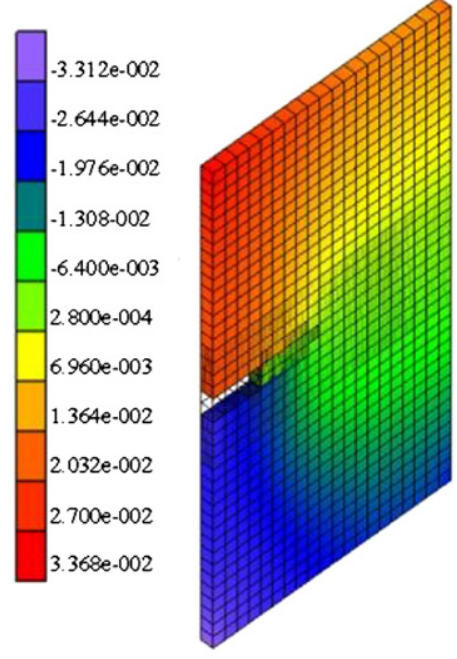

(c) step $=\mathbf{3 0}$
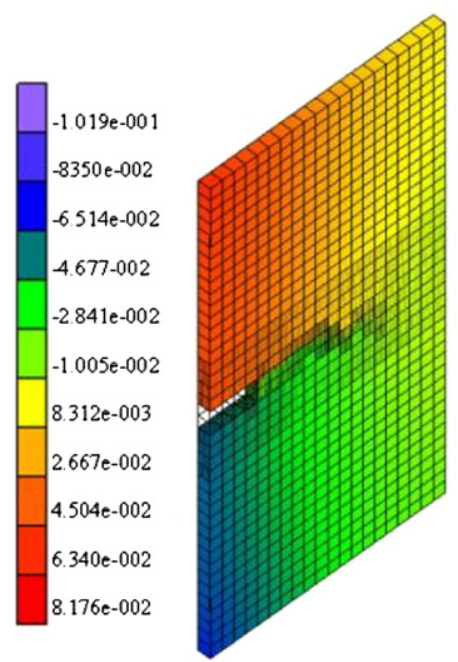

(f) $\operatorname{step}=60$

Fig. 19. Contour map of the $y$-direction displacement at the different steps.

\subsection{Releasing PMM element into DLSM}

In this section, we will discuss the technique of automatically releasing the PMM element into the DLSM during numerical simulations. The release of particles is treated as a pre-failure process in the m-DLSM. A reduced macroscopic strength criterion is used as the releasing criterion. When the state of the PMM element satisfies this criterion, the PMM element is transformed into the DLSM model. In this paper, a simple maximum strain-based criterion is used as the releasing rule. The PMM element will be released into the DLSM model when strain state of the PMM element satisfies

$\varepsilon_{1}>\gamma \varepsilon_{t}^{*}$

where $\varepsilon_{1}$ is the maximum main strain of the PMM element, $\varepsilon_{t}^{*}$ is the ultimate strain of the model and $\gamma$ is a reduction factor which is taken as 0.8 . When the PMM element is released, it will be removed from the calculation cycle and newly released particles will take part in the subsequent calculation cycle of the DLSM model. The failure in the DLSM is realized: when the normal or shear displacement of the bond exceeds a prescribed value. In this case, the bond between to particles is broken and a contact bond with zero strength is established.

\section{Examples}

\subsection{Simple tensional test}

In this section, the pure tensile loading of a bar measuring $10 \mathrm{~mm} \times 10 \mathrm{~mm} \times 20 \mathrm{~mm}$ is simulated. The purpose is to test the influence of the different coupling techniques on the simulation results and to validate the correctness of the proposed method. Four coupled DLSM models are shown in Fig. 9. The applied boundary force is $1 \mathrm{MPa}$ and the elastic properties of the model are as follows: elastic modulus $12.5 \mathrm{GPa}$ and Poisson's ratio 0.3. The first model is a full DLSM model (see Fig. 9a) which is made up of $1 \mathrm{~mm}$ diameter particles; the second is made up of the DLSM model and the PMM models with an element length of $5 \mathrm{~mm}$ (Fig. 9b). The third model uses the NMM \& DLSM (see Fig. 9c), and the final model is a three layer model that includes the NMM, PMM \& DLSM models (shown in Fig. 9d). The simulation results in terms of contour maps of displacement in the z-direction (loaded direction) are shown in Fig. 10.

From Fig. 10 it can be seen that the DLSM \& NMM without appropriate coupling cannot predict the displacement pattern correctly (see Fig. 10c). The results are improved when DLSM \& NMM 
models are coupled using the proposed PMM model. A comparison of the displacements in the $z$-direction at the top surface of the model are compared with the analytical solution. The expected displacement in the $\mathrm{z}$ direction of the top surface is given as

$u^{*}=\frac{\left(1-v^{2}\right) P L^{*}}{E}$

where $u^{*}$ is the expected displacement and $L^{*}$ is the effective length of the model which is defined as the distance from the fixed boundary to the loading point. Notice that for particle based models, due to the size of the particles and the fact that we can only apply the boundary conditions at the center of the particles, therefore, the effective length for different coupled models may not be identical depending on the configuration of the particle based component of the model. The effective length and the predicted displacement in $z$-direction for the different coupled DLSM models are listed in Table 1, which shows that the appropriateness of the proposed coupling procedure.

To further verity the application of model proposed, the problem of a cantilever beam is simulated using the DLSM \& PMM \& NMM coupled model (Fig. 9d). The left end of the beam is fixed and the right end is subjected to a traction force of $1 \mathrm{MPa}$. The particle size is taken as $0.25 \mathrm{~mm}$ in diameter and the mesh size of the NMM model is $1.0 \mathrm{~mm} \times 1.0 \mathrm{~mm} \times 1.0 \mathrm{~mm}$. The problem is also simulated using a FEM model with a resolution of $10 \times 10 \times 40$ using 40008 -node elements, as a reference solution. Two section lines, Line I $((2.375,2.375,10.125)-(2.375,2.375,19.875))$ and Line II $((7.375,7.375,10.125)-(7.375,7.375,19.875))$, are selected for recording of the displacements predicted by the coupled DLSM model. A full comparison with the displacement field of the FEM is given in Fig. 11. It can be seen that the displacement field predicted by coupled DLSM is almost identical to that of FEM model. The maximum error for the displacement in the y-direction, along these two lines, is less than $2.00 \%$.

\subsection{Uniaxial loading of a plate with a circular hole}

A square plate containing a central circular hole is selected as another example to demonstrate the ability of coupled DLSM to model static elastic problems. The dimension of the plate is $100 \mathrm{~mm} \times 200 \mathrm{~mm} \times 10 \mathrm{~mm}$ and a circular hole with radius of $20 \mathrm{~mm}$ is located at the center of the plate. The two coupled DLSM models used are shown in Fig. 12, the DLSM \& PMM \& NMM model (Fig. 12a) and PMM \& NMM model (Fig. 12b). The applied boundary force at the top of the plane is $1 \mathrm{MPa}$ and the bottom boundary is fixed during calculation. Material properties of the model are the elastic modulus, $12.5 \mathrm{Gpa}$, and the Poisson's ratio, 0.30 .
Contour maps of the displacement in the $y$-direction for these two models are shown in Fig. 13. The same distributions are obtained for different coupled models, showing that the PMM model can reproduce the DLSM results with a substantially reduced computational effort. The displacements in the $y$-direction at detection points, A $(0.5,100.5,5.5), \mathrm{B}(10.5,100.5,5.5), \mathrm{C}(20.5,100.5,5.5)$, D (79.5, 100.5, 5.5), E (89.5, 100.5, 5.5) and $\mathrm{F}(99.5,100.5,5.5)$, were recorded and are listed in Table 2 .

\subsection{Wave propagation through an elastic bar}

This example is used to show the ability of the coupled DLSM to model wave propagation through an elastic bar. The two coupled DLSM models are shown in Fig. 14. The model dimensions are $20 \mathrm{~mm} \times 20 \mathrm{~mm} \times 200 \mathrm{~mm}$. The material parameters are: elastic modulus $12.5 \mathrm{GPa}$, Poisson's ratio 0.3 and density $2650 \mathrm{~kg} / \mathrm{m}^{3}$. A half-cycle sinusoidal velocity wave with $100 \mathrm{~mm} / \mathrm{s}$ amplitude and $50,000 \mathrm{~Hz}$ frequency is applied at the left boundary. The right boundary of the bar is free while the other four side boundaries are all fixed in their normal direction.

Fig. 15 show the contour maps of the particle velocity in the $z$ direction for the full DLSM model and DLSM \& PMM coupled model. The propagation and reflection of the wave can be observed clearly for these two models. Four detection points, A $(5.5,5.5$, $0.5), \mathrm{B}(5.5,5.5,50.5), \mathrm{C}(5.5,5.5,150.5)$ and $\mathrm{D}(5.5,5.5,199.5)$, are placed in the bar to record the wave propagation through the model. The recorded waves at these points for the different models

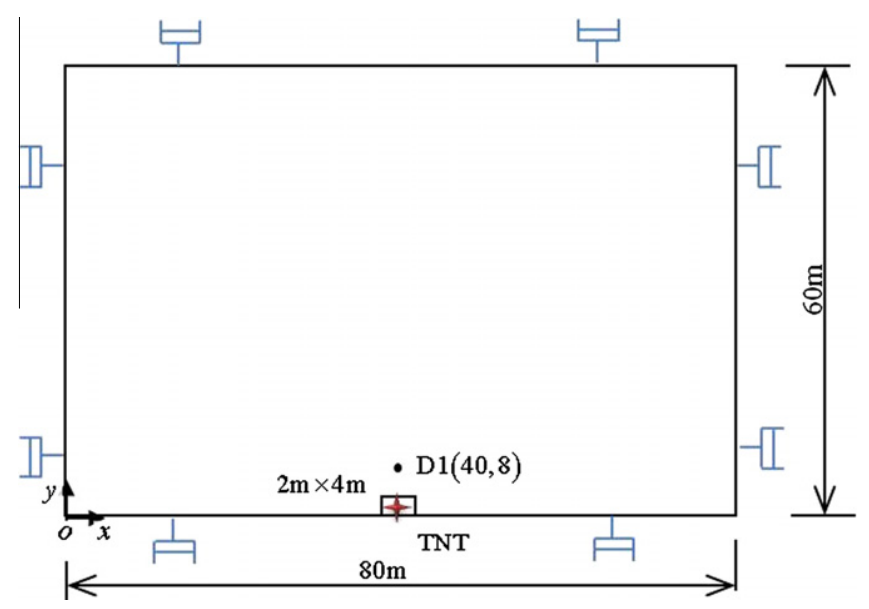

Fig. 20. The computational model of blasting wave propagation through rock cavern.

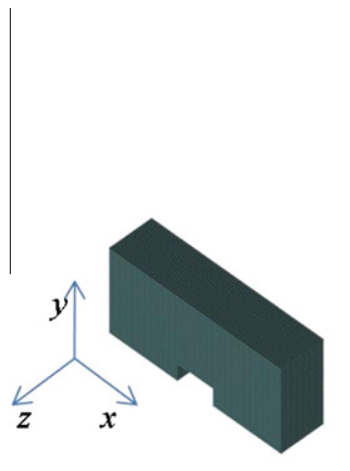

(a) DLSM model

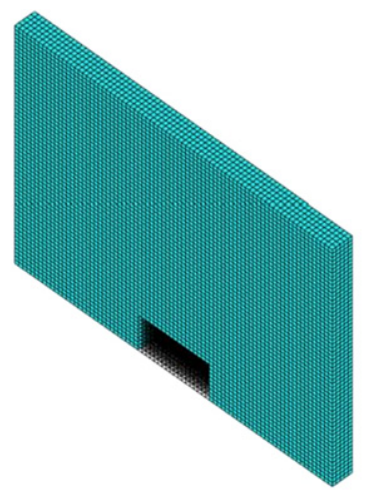

(b) NMM model

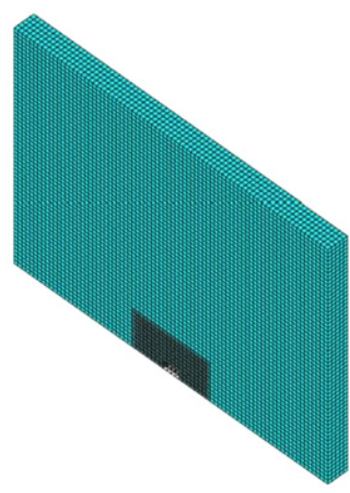

(c) Coupled model

Fig. 21. The coupled model for the blast wave propagation through rock cavern. 


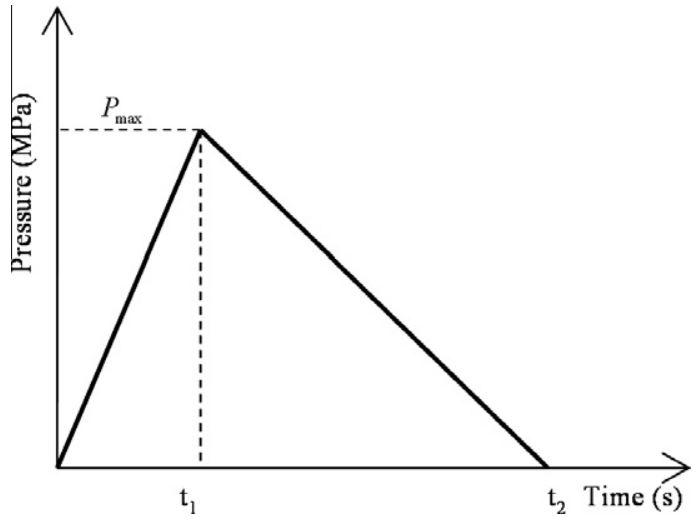

Fig. 22. Triangle pressure wave to represent blasting loading.

are shown in Fig. 16. The DLSM \& PMM model produces slightly different wave forms at some detection points since the PMM element size is larger than the particle size of the DLSM which causes some high frequency parts of the wave to be filtered out in the coupled DLSM \& PMM model. Even so, both the wave form and the amplitude are in good agreement between two models. This example shows that coupled DLSM can accurately predict the dynamic loads transmitted through an elastic body.

\subsection{Progressive failure of a solid specimen with a side notch}

A solid specimen with a side notch, as shown in Fig. 17, is simulated using the m-DLSM. The mechanical properties of the material are the elastic modulus $12.5 \mathrm{GPa}$, Poisson's ratio 0.3 and density $2650 \mathrm{~kg} / \mathrm{m}^{3}$. The particle size of the DLSM model is taken as $1 \mathrm{~mm}$ and the manifold element length is taken as $5 \mathrm{~mm}$. The solid specimen is $100 \mathrm{~mm} \times 200 \mathrm{~mm} \times 5 \mathrm{~mm}$ and the notch is $20 \mathrm{~mm} \times 5 \mathrm{~mm} \times 5 \mathrm{~mm}$. The ultimate strain for the PMM element is taken as $4 \times 10^{-4}$ and the reduction factor for the releasing criteria is taken as 0.8 . The ultimate deformation of the lattice bond in the DLSM is given as $5 \times 10^{-4} \mathrm{~mm}$. The applied force on the top boundary is taken as $1 \mathrm{MPa}$ while the bottom boundary is fixed.

During computation, the PMM elements near the notch are first transformed into the DLSM model. The DLSM is then further broken down and finally forms a fracture. Fig. 18 shows the process of PMM elements releasing/converting into DLSM particles. Contour maps of the $y$-displacement for the six stages are presented in Fig. 19. With regard to the failure patterns obtained, the simulation gives a realistic description of the fracturing process of the notched solid specimen under tensile loading, which is only

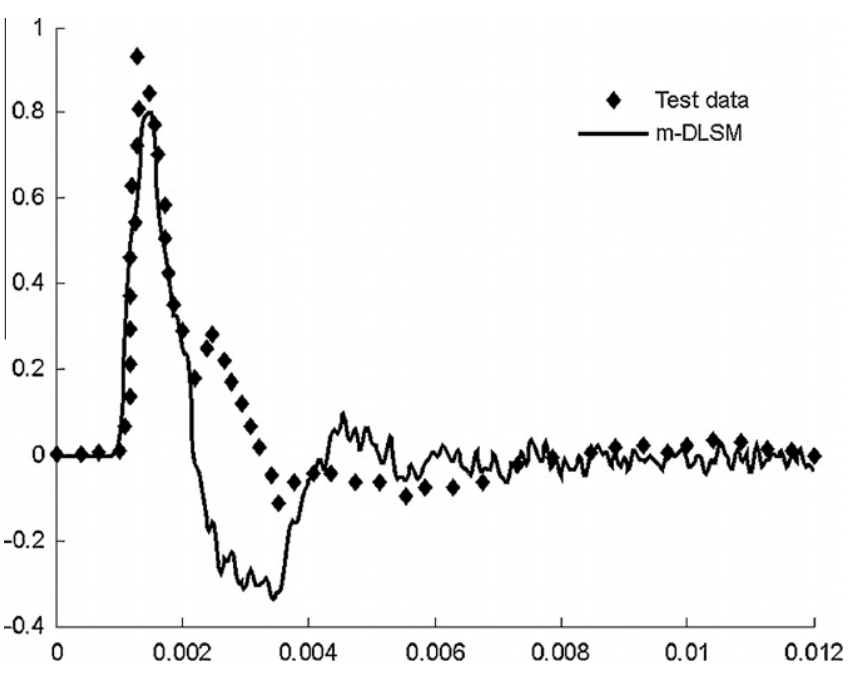

Fig. 23. The velocity histories predicted by coupled DLSM and field test.

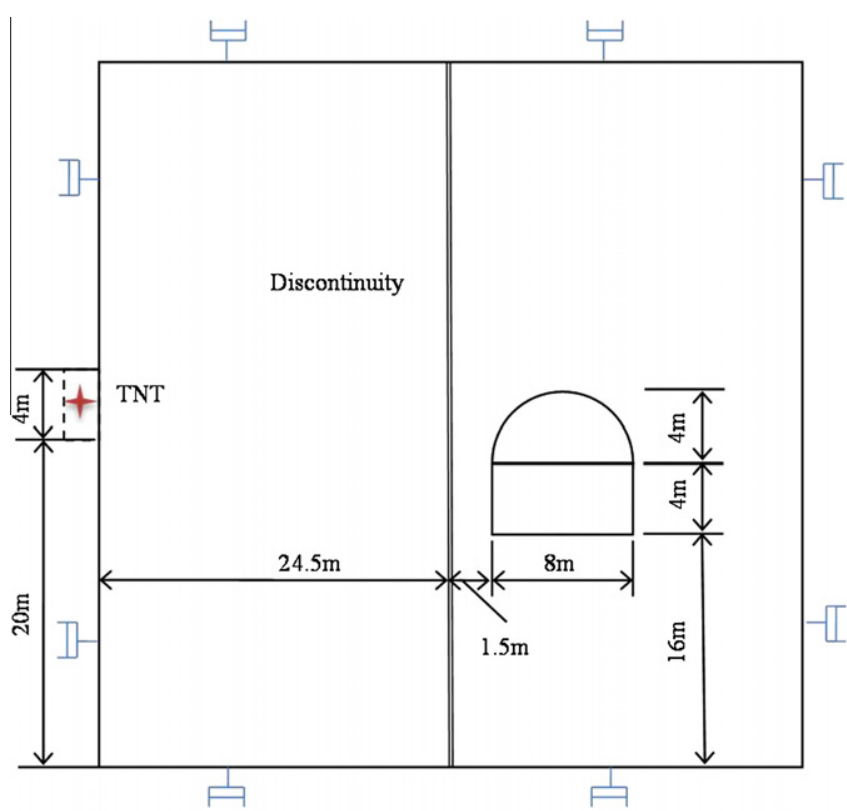

Fig. 24. Computational model for a tunnel under blast loading.

a simple example to show the ability of the proposed method to model crack propagation.

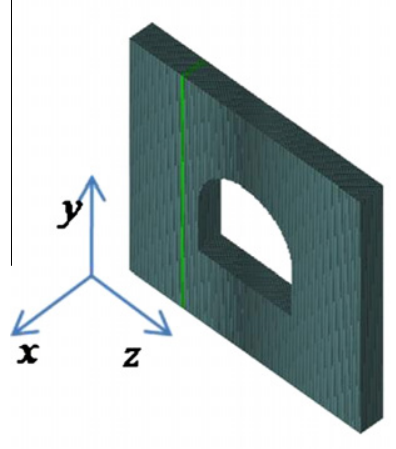

(a) DLSM model

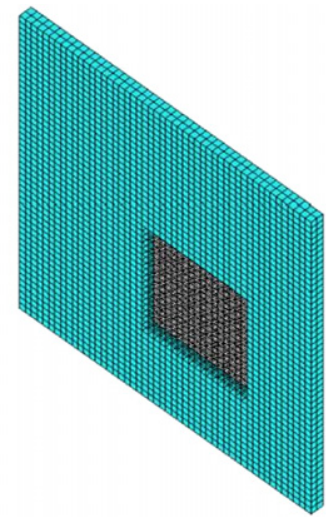

(b) NMM model

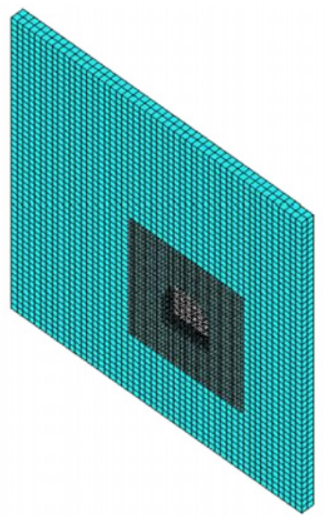

(c) m-DLSM model

Fig. 25. The coupled model for the blast wave propagation through a tunnel. 

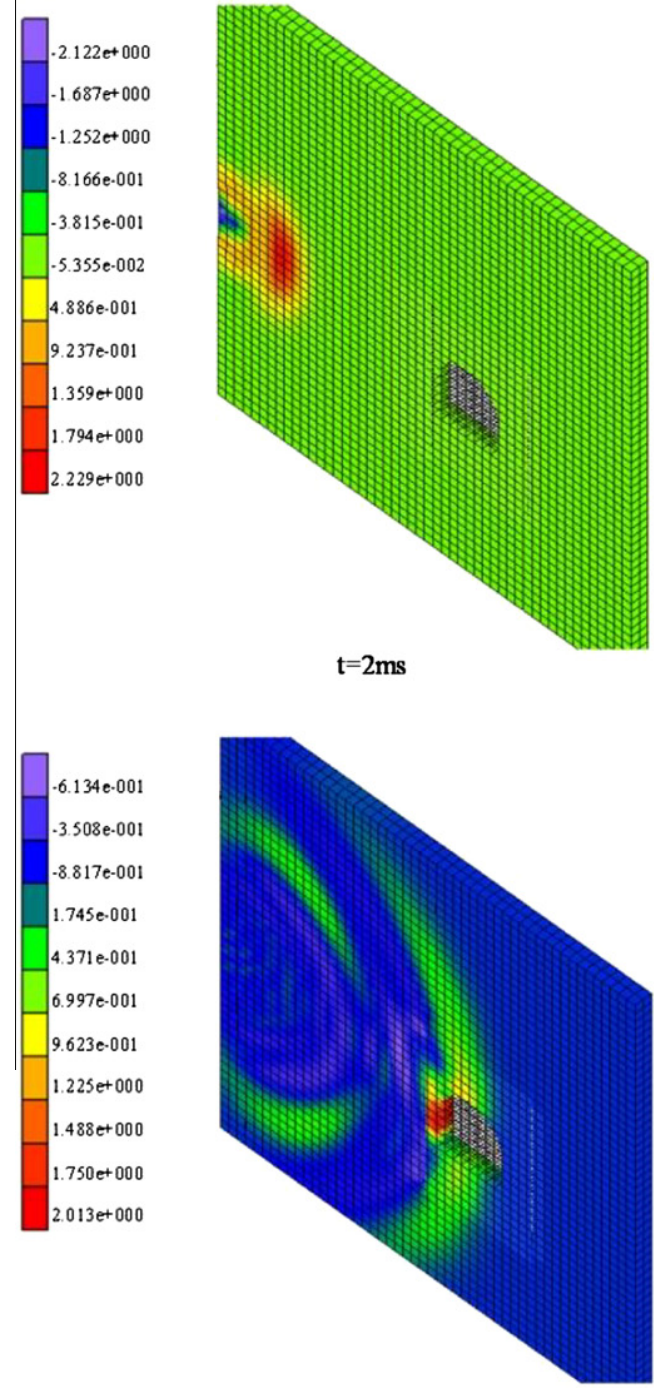

$\mathrm{t}=6 \mathrm{~ms}$
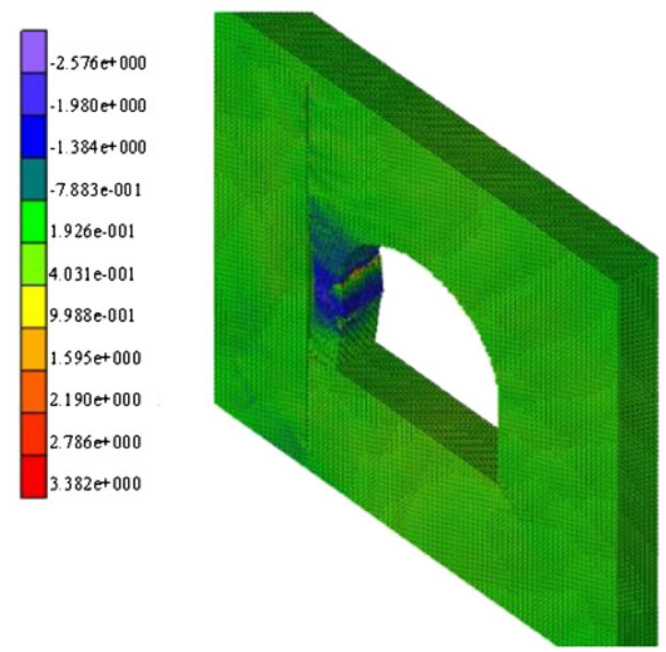

$\mathrm{t}=10 \mathrm{~ms}$
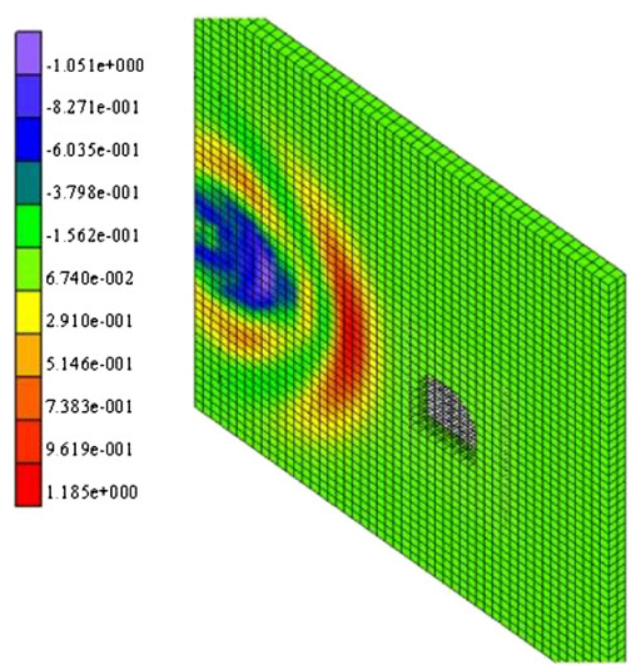

$t=4 \mathrm{~ms}$
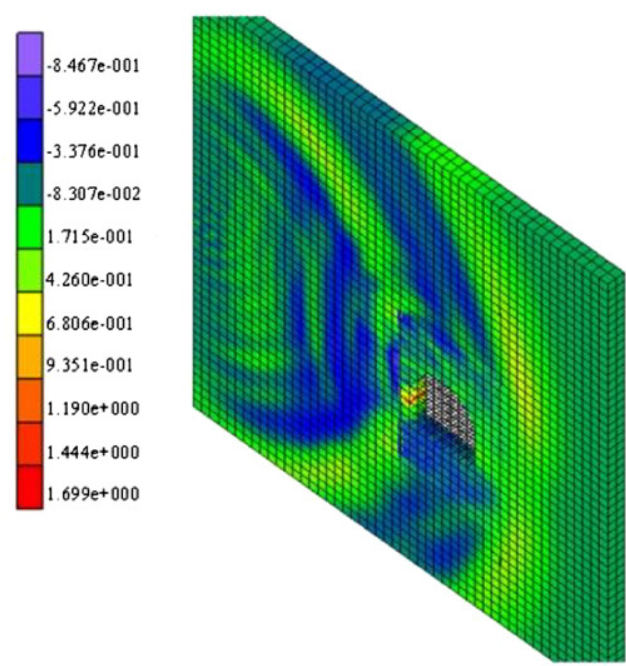

$\mathrm{t}=\mathbf{8} \mathrm{ms}$
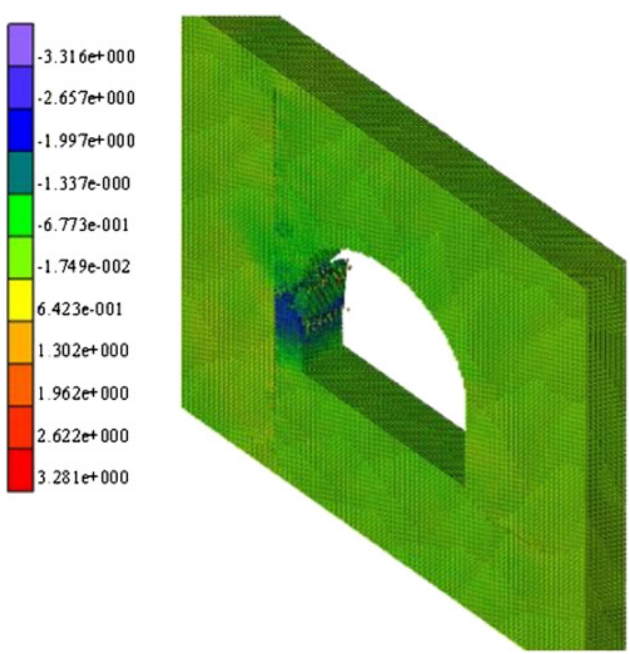

$\mathrm{t}=12 \mathrm{~ms}$

Fig. 26. The failure process of a tunnel under blast loading (Model III) (contour map of velocity in $x$-direction). 


\subsection{Dynamic failure of a tunnel under blasting loading}

\subsubsection{Blasting wave propagation through rock cavern}

In this section, the blasting induced wave propagation through rock cavern is simulated by coupled DLSM and compared with the field test data provided in $[31,32]$. The computational model with dimension of $80 \mathrm{~m} \times 60 \mathrm{~m} \times 5 \mathrm{~m}$ is constructed, where an explosion chamber of $4 \mathrm{~m} \times 2 \mathrm{~m}$ is excavated (seen in Fig. 20). The coupled DLSM is shown in Fig. 21, the particle size used is $0.25 \mathrm{~m}$ in diameter and the mesh size of the NMM model is given as $1.00 \mathrm{~m} \times 1.00 \mathrm{~m} \times 1.00 \mathrm{~m}$. A plane strain analysis is performed. The mechanical properties assumed for the rock material are: the elastic modulus $=74.0 \mathrm{GPa}$, the Poisson's ratio $=0.25$ and the density $=2650 \mathrm{~kg} / \mathrm{m}^{3}$, which are typical of the material parameters for Bukit Timah granite [31]. A triangular over-pressure history with two phases (see Fig. 22) is used to represent the blasting wave of effective TNT charge weight of $606 \mathrm{~kg}$ with a loading density of $10 \mathrm{~kg} / \mathrm{m}^{3}$. The maximum over-pressure $P_{\max }$ is equal to $30.23 \mathrm{MPa}$, the duration of rise phase $t_{1}$ and the total duration $t_{2}$ are 0.5 and $2.5 \mathrm{~ms}$, respectively. These parameters of triangular over-pressure history are calculated using the empirical equations provided in [33]. The velocity history predicted by coupled DLSM model is compared with the test data at $8 \mathrm{~m}$ above the detonation point (see Fig. 23). The agreement of DLSM modeling and field test is similar with the results of FEM reported in [33].

\subsection{Dynamic failure of a tunnel under blasting loading}

Here, the blast wave propagation through a rock mass and the influence of discontinuities on the failure pattern of a tunnel is simulated using the m-DLSM, which is a problem of tremendous concern in rock engineering [34,35]. Fig. 24 shows the computa-

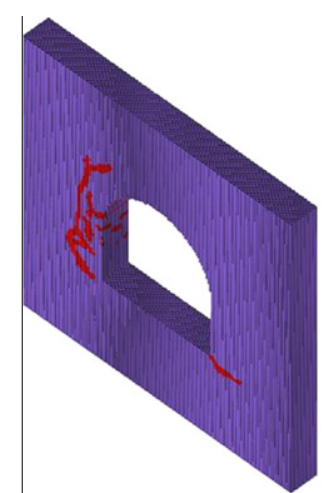

(a) Model I

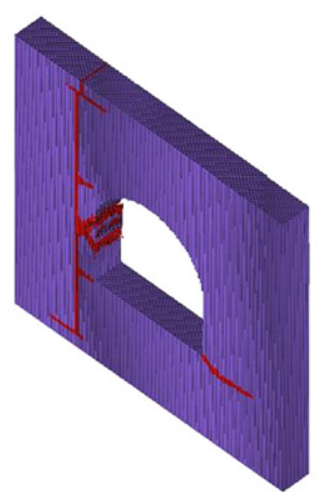

(c) Model III

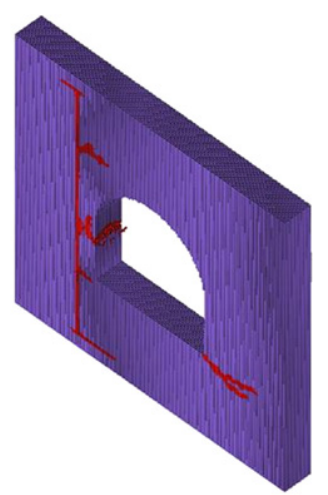

(b) Model II

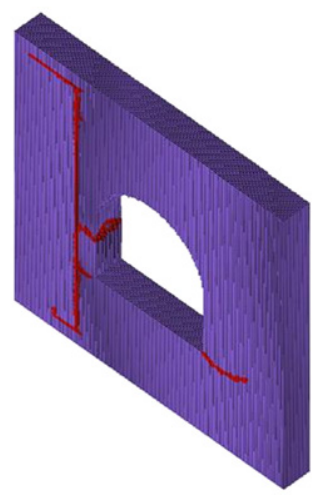

(d) Model IV
Fig. 27. Failure modes of different models under blasting loads tional model and boundary conditions of the problem analysed. The model is $50 \mathrm{~m} \times 50 \mathrm{~m} \times 2 \mathrm{~m}$ and with a particle size of $0.125 \mathrm{~m}$. For the DLSM model, more than two million particles are needed to build the computational model; that is more than ten millions of pieces of bond information needs to be stored, which is computationally prohibitive for a normal PC. However, only about half a million particles are used in the coupled DLSM model (see Fig. 25). A blasting load is applied at the left of the boundary from $20 \mathrm{~m}$ to $24 \mathrm{~m}$ vertically to simulate an explosion chamber of $4 \mathrm{~m} \times 2 \mathrm{~m}$. The blast wave is inputted as a triangular over-pressure history with two phases exactly as in the previous section. The material properties of the rock are: elastic modulus $74 \mathrm{GPa}$, Poisson's ratio 0.2 and density $2650 \mathrm{~kg} / \mathrm{m}^{3}$. The ultimate bond deformation is $2.5 \mathrm{e}-6 \mathrm{~m}$. A discontinuity is represented by setting a material layer with a weaker elastic modulus, where the weakness ratio is taken as 1.0 (Model I), 0.5 (Model II), 0.1 (Model III) and 0.01 (Model IV). The modeling results of Model III are shown in Fig. 26, where the left hand side of the tunnel is broken under blast loading.

The failure patterns of coupled DLSM models with different stiffnesses of discontinuity are shown in Fig. 27. The failure pattern of the tunnel is influenced by the stiffness of the discontinuity. When the stiffness is decreased, the degree of damage first increases and then decreases. This is an interesting result and shows that the damage of a tunnel under dynamic loading can be reduced through pre-setting some weak discontinuity/cavern. If the discontinuity is weak enough spalling happens further away from the tunnel (see Fig. 27d). This example clearly shows that the coupled DLSM can be used to solve previously inaccessible problems using DLSM in a normal PC. However, more complex constitutive modeling for bond springs in DLSM and more advanced representation techniques of discontinuities are needed to further apply the coupled DLSM to real engineering problems.

\section{Conclusions}

This paper presents a coupled lattice spring model, in which the DLSM is coupled with the NMM. A three layer structure is used to combine DLSM and NMM, using the PMM to bridge the DLSM and NMM. PMM simplifies contact detection between the particles in DLSM model and the NMM model and also serves as a cushioning layer which can reduce the spurious wave reflection between the micro and macro models. The proposed coupled model can be used to model dynamic fracturing and wave propagation problems. A few examples are provided to validate the correctness of the proposed coupling method. The examples given highlight the potential of the coupled DLSM in solving problems previously inaccessible for DLSM using a normal PC.

\section{Acknowledgments}

The authors thank the anonymous reviewer for the valuable comments and helpful suggestions.

\section{Appendix A}

In this appendix, Eq. (7) used for evaluating the deformation of shear springs in DLSM is derived. Consider a cubical unit volume containing a bond connecting two particles as shown in Fig. A1.

The complete 1st order displacement function of the cube is

$$
\left(\begin{array}{l}
u_{x} \\
u_{y} \\
u_{z}
\end{array}\right)=\left(\begin{array}{llll}
a_{0} & a_{1} & a_{2} & a_{3} \\
b_{0} & b_{1} & b_{2} & b_{3} \\
c_{0} & c_{1} & c_{2} & c_{3}
\end{array}\right)\left(\begin{array}{l}
1 \\
x \\
y \\
z
\end{array}\right)
$$


Assuming the center of the cube is at $\left(x_{c}, y_{c}, z_{c}\right)$, then its displacement is represented by

$$
\left(\begin{array}{l}
u_{c x} \\
u_{c y} \\
u_{c z}
\end{array}\right)=\left(\begin{array}{llll}
a_{0} & a_{1} & a_{2} & a_{3} \\
b_{0} & b_{1} & b_{2} & b_{3} \\
c_{0} & c_{1} & c_{2} & c_{3}
\end{array}\right)\left(\begin{array}{c}
1 \\
x_{c} \\
y_{c} \\
z_{c}
\end{array}\right)
$$

Subtracting (A.2) from (A.1) gives

$$
\begin{aligned}
\left(\begin{array}{l}
u_{x} \\
u_{y} \\
u_{z}
\end{array}\right)-\left(\begin{array}{l}
u_{c x} \\
u_{c y} \\
u_{c z}
\end{array}\right)= & \left(\begin{array}{llll}
a_{0} & a_{1} & a_{2} & a_{3} \\
b_{0} & b_{1} & b_{2} & b_{3} \\
c_{0} & c_{1} & c_{2} & c_{3}
\end{array}\right)\left(\begin{array}{l}
1 \\
x \\
y \\
z
\end{array}\right) \\
& -\left(\begin{array}{llll}
a_{0} & a_{1} & a_{2} & a_{3} \\
b_{0} & b_{1} & b_{2} & b_{3} \\
c_{0} & c_{1} & c_{2} & c_{3}
\end{array}\right)\left(\begin{array}{c}
1 \\
x_{c} \\
y_{c} \\
z_{c}
\end{array}\right)
\end{aligned}
$$

Equation (A.3) can be further written as

$$
\begin{aligned}
\left(\begin{array}{l}
u_{x} \\
u_{y} \\
u_{z}
\end{array}\right)= & \left(\begin{array}{l}
u_{c x} \\
u_{c y} \\
u_{c z}
\end{array}\right)+\left(\begin{array}{ccc}
a_{1} & 0 & 0 \\
0 & b_{2} & 0 \\
0 & 0 & c_{3}
\end{array}\right)\left(\begin{array}{l}
x-x_{c} \\
y-y_{c} \\
z-z_{c}
\end{array}\right) \\
& +\left(\begin{array}{ccc}
0 & a_{2} & a_{3} \\
b_{1} & 0 & b_{3} \\
c_{1} & c_{2} & 0
\end{array}\right)\left(\begin{array}{l}
x-x_{c} \\
y-y_{c} \\
z-z_{c}
\end{array}\right)
\end{aligned}
$$

From (A.4), we have

$$
\begin{aligned}
\varepsilon_{x x}=\frac{\partial u_{x}}{\partial x}=a_{1} \\
\varepsilon_{y y}=\frac{\partial u_{y}}{\partial y}=b_{2} \\
\varepsilon_{z z}=\frac{\partial u_{z}}{\partial z}=c_{3} \\
\omega_{x}=\frac{1}{2}\left(\frac{\partial u_{z}}{\partial y}-\frac{\partial u_{y}}{\partial z}\right)=\frac{1}{2}\left(c_{2}-b_{3}\right) \\
\omega_{y}=\frac{1}{2}\left(\frac{\partial u_{x}}{\partial z}-\frac{\partial u_{z}}{\partial x}\right)=\frac{1}{2}\left(a_{3}-c_{1}\right) \\
\omega_{z}=\frac{1}{2}\left(\frac{\partial u_{y}}{\partial x}-\frac{\partial u_{x}}{\partial y}\right)=\frac{1}{2}\left(b_{1}-a_{2}\right) \\
\varepsilon_{y z}=\frac{1}{2}\left(\frac{\partial u_{z}}{\partial y}+\frac{\partial u_{y}}{\partial z}\right)=\frac{1}{2}\left(c_{2}+b_{3}\right) \\
\varepsilon_{z x}=\frac{1}{2}\left(\frac{\partial u_{x}}{\partial z}+\frac{\partial u_{z}}{\partial x}\right)=\frac{1}{2}\left(a_{3}+c_{1}\right) \\
\varepsilon_{x y}=\frac{1}{2}\left(\frac{\partial u_{y}}{\partial x}+\frac{\partial u_{x}}{\partial y}\right)=\frac{1}{2}\left(b_{1}+a_{2}\right)
\end{aligned}
$$

Using the above relations, Eq. (A.4) can be transformed into

$$
\left(\begin{array}{l}
u_{x} \\
u_{y} \\
u_{z}
\end{array}\right)=\left[\mathbf{D}_{\mathbf{0}}\right]\left(\begin{array}{l}
u_{c x} \\
u_{c y} \\
u_{c z}
\end{array}\right)+\left[\mathbf{D}_{1}\right]\left(\begin{array}{c}
\omega_{x} \\
\omega_{y} \\
\omega_{z}
\end{array}\right)+\left[\mathbf{D}_{2}\right]\left(\begin{array}{c}
\varepsilon_{x x} \\
\varepsilon_{y y} \\
\varepsilon_{z z}
\end{array}\right)+\left[\mathbf{D}_{3}\right]\left(\begin{array}{c}
\varepsilon_{y z} \\
\varepsilon_{z x} \\
\varepsilon_{x y}
\end{array}\right)
$$

where

$$
\begin{aligned}
& {\left[\mathbf{D}_{0}\right]=\left(\begin{array}{lll}
1 & 0 & 0 \\
0 & 1 & 0 \\
0 & 0 & 1
\end{array}\right)} \\
& {\left[\mathbf{D}_{1}\right]=\left(\begin{array}{ccc}
0 & z-z_{c} & -\left(y-y_{c}\right) \\
-\left(z-z_{c}\right) & 0 & x-x_{c} \\
y-y_{c} & -\left(x-x_{c}\right) & 0
\end{array}\right)} \\
& {\left[\mathbf{D}_{2}\right]=\left(\begin{array}{ccc}
x-x_{c} & 0 & 0 \\
0 & y-y_{c} & 0 \\
0 & 0 & z-z_{c}
\end{array}\right)} \\
& {\left[\mathbf{D}_{3}\right]=\left(\begin{array}{ccc}
0 & z-z_{c} & y-y_{c} \\
z-z_{c} & 0 & x-x_{c} \\
y-y_{c} & x-x_{c} & 0
\end{array}\right)}
\end{aligned}
$$

Denoting the coordinates of the two particles in the cube as $\left(x_{1}, y_{1}\right.$, $\left.z_{1}\right)$ and $\left(x_{2}, y_{2}, z_{2}\right)$ and the displacement of particles as $\left(u_{1}, v_{1}, w_{1}\right)$ and $\left(u_{2}, v_{2}, w_{2}\right)$, the relative displacement vector between the two particles is

$$
\left(\begin{array}{l}
u_{12 x} \\
u_{12 y} \\
u_{12 z}
\end{array}\right)=\left(\begin{array}{ll}
u_{2 x} & -u_{1 x} \\
u_{2 y} & -u_{1 y} \\
u_{2 z} & -u_{1 z}
\end{array}\right)
$$

and the normal unit vector is

$$
\left(\begin{array}{l}
n_{x} \\
n_{y} \\
n_{z}
\end{array}\right)=\left(\begin{array}{c}
\frac{x_{2}-x_{1}}{l} \\
\frac{y_{2}-y_{1}}{l} \\
\frac{z_{2}-z_{1}}{l}
\end{array}\right)
$$

where $l$ is the length of the bond. The relative normal displacement vector is defined as

$$
\left(\begin{array}{l}
u_{12 x}^{n} \\
u_{12 y}^{n} \\
u_{12 z}^{n}
\end{array}\right)=\left(\left(\begin{array}{l}
u_{12 x} \\
u_{12 y} \\
u_{12 z}
\end{array}\right)^{T}\left(\begin{array}{l}
n_{x} \\
n_{y} \\
n_{z}
\end{array}\right)\right)\left(\begin{array}{l}
n_{x} \\
n_{y} \\
n_{z}
\end{array}\right)
$$

By vector operation, the relative shear displacement vector is obtained as

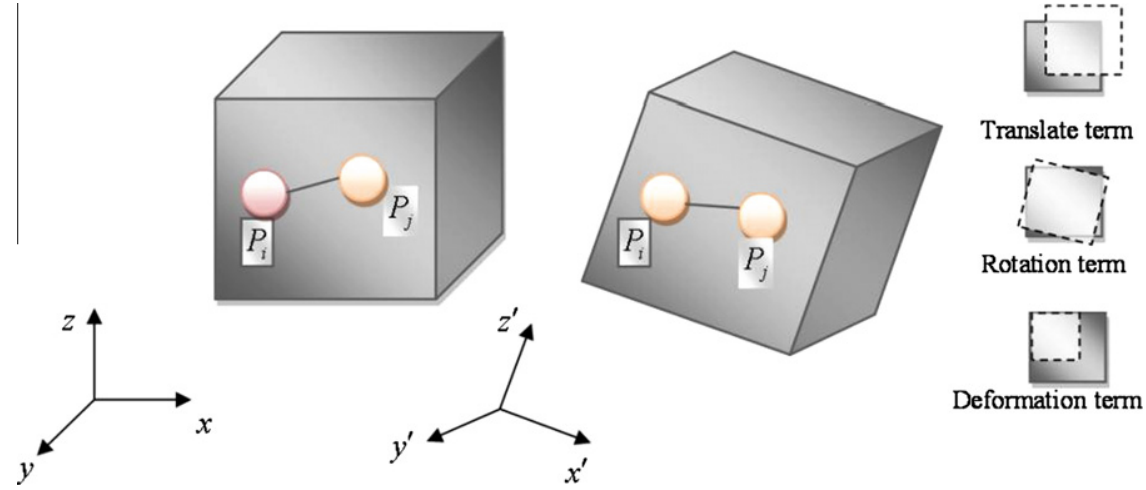

Fig. A1. Illustration of the deformation of a cubic unit with a bond connecting two particles. 


$$
\begin{aligned}
\left(\begin{array}{l}
u_{12 x}^{s} \\
u_{12 y}^{s} \\
u_{12 z}^{s}
\end{array}\right) & =\left(\begin{array}{l}
u_{12 x} \\
u_{12 y} \\
u_{12 z}
\end{array}\right)-\left(\begin{array}{l}
u_{12 x}^{n} \\
u_{12 y}^{n} \\
u_{12 z}^{n}
\end{array}\right) \\
& =\left(\begin{array}{l}
u_{12 x} \\
u_{12 y} \\
u_{12 z}
\end{array}\right)-\left(\left(\begin{array}{l}
u_{12 x} \\
u_{12 y} \\
u_{12 z}
\end{array}\right)^{T}\left(\begin{array}{l}
n_{x} \\
n_{y} \\
n_{z}
\end{array}\right)\right)\left(\begin{array}{l}
n_{x} \\
n_{y} \\
n_{z}
\end{array}\right)
\end{aligned}
$$

Now, applying the equation (A.5), the relative displacement vector can be represented as

$$
\begin{aligned}
\left(\begin{array}{ll}
u_{2 x} & -u_{1 x} \\
u_{2 y} & -u_{1 y} \\
u_{2 z} & -u_{1 z}
\end{array}\right)= & \underbrace{\left(\begin{array}{ccc}
x_{2}-x_{1} & 0 & 0 \\
0 & y_{2}-y_{1} & 0 \\
0 & 0 & z_{2}-z_{1}
\end{array}\right)\left(\begin{array}{l}
\varepsilon_{x x} \\
\varepsilon_{y y} \\
\varepsilon_{z z}
\end{array}\right)+\left(\begin{array}{ccc}
0 & z_{2}-z_{1} & y_{2}-y_{1} \\
z_{2}-z_{1} & 0 & x_{2}-x_{1} \\
y_{2}-y_{1} & x_{2}-x_{1} & 0
\end{array}\right)\left(\begin{array}{l}
\varepsilon_{y z} \\
\varepsilon_{z x} \\
\varepsilon_{x y}
\end{array}\right)}_{\text {strain related term }} \\
& +\underbrace{\left(\begin{array}{ccc}
0 & z_{2}-z_{1} & -\left(y_{2}-y_{1}\right) \\
-\left(z_{2}-z_{1}\right) & 0 & x_{2}-x_{1} \\
y_{2}-y_{1} & -\left(x_{2}-x_{1}\right) & 0
\end{array}\right)\left(\begin{array}{c}
\omega_{x} \\
\omega_{y} \\
\omega_{z}
\end{array}\right)}_{\text {rotation related term }}
\end{aligned}
$$

With the above equation, it is straightforward to show that the relative normal displacement vector is only dependant on the corresponding strain term because of the following equivalence

$$
\left(\begin{array}{ccc}
0 & z_{2}-z_{1} & -\left(y_{2}-y_{1}\right) \\
-\left(z_{2}-z_{1}\right) & 0 & x_{2}-x_{1} \\
y_{2}-y_{1} & -\left(x_{2}-x_{1}\right) & 0
\end{array}\right)\left(\begin{array}{l}
x_{2}-x_{1} \\
y_{2}-y_{1} \\
z_{2}-z_{1}
\end{array}\right)=0
$$

However, for the relative shear displacement vector, if we directly substitute (A.10) into (A.9), the term due to rotation will not vanish. It is known that rigid rotation of the cube should not produce strain energy. Therefore, in DLSM, the rotation related term is removed from the calculation of the relative shear displacement vector, namely, the relative displacement vector in (A.9) is not calculated

\begin{tabular}{|c|c|c|}
\hline$i$ & $\begin{array}{l}N_{i}(x, y, z)=1-\frac{\left(x-x_{i}\right)\left(y-y_{i}\right)\left(z-z_{i}\right)}{L^{3}} \\
N_{i, y}(x, y, z)=-\frac{\left(x-x_{i}\right)\left(z-z_{i}\right)}{L^{3}}\end{array}$ & $\begin{array}{l}N_{i, x}(x, y, z)=-\frac{\left(y-y_{i}\right)\left(z-z_{i}\right)}{L^{3}} \\
N_{i, z}(x, y, z)=-\frac{\left(x-x_{i}\right)\left(y-y_{i}\right)}{L^{3}}\end{array}$ \\
\hline$j$ & $\begin{array}{l}N_{j}(x, y, z)=1+\frac{\left(x-x_{j}\right)\left(y-y_{j}\right)\left(z-z_{j}\right)}{L^{3}} \\
N_{i, y}(x, y, z)=-\frac{\left(x-x_{i}\right)\left(z-z_{i}\right)}{L^{3}}\end{array}$ & $\begin{array}{l}N_{j, x}(x, y, z)=\frac{\left(y-y_{j}\right)\left(z-z_{j}\right)}{L^{3}} \\
N_{i, z}(x, y, z)=-\frac{\left(x-x_{i}\right)\left(y-y_{i}\right)}{L^{3}}\end{array}$ \\
\hline$k$ & $\begin{array}{l}N_{k}(x, y, z)=1-\frac{\left(x-x_{k}\right)\left(y-y_{k}\right)\left(z-z_{k}\right)}{L^{3}} \\
N_{k, y}(x, y, z)=-\frac{\left(x-x_{k}\right)\left(z-z_{k}\right)}{L^{3}}\end{array}$ & $\begin{array}{l}N_{k, x}(x, y, z)=-\frac{\left(y-y_{k}\right)\left(z-z_{k}\right)}{L^{3}} \\
N_{k, z}(x, y, z)=-\frac{\left(x-x_{k}\right)\left(y-y_{k}\right)}{L^{3}}\end{array}$ \\
\hline$l$ & $\begin{array}{l}N_{l}(x, y, z)=1+\frac{\left(x-x_{l}\right)\left(y-y_{l}\right)\left(z-z_{l}\right)}{L^{3}} \\
N_{l, y}(x, y, z)=\frac{\left(x-x_{l}\right)\left(z-z_{l}\right)}{L^{3}}\end{array}$ & $\begin{array}{l}N_{l, x}(x, y, z)=\frac{\left(y-y_{l}\right)\left(z-z_{l}\right)}{L^{3}} \\
N_{l, z}(x, y, z)=\frac{\left(x-x_{l}\right)\left(y-y_{l}\right)}{L^{3}}\end{array}$ \\
\hline$m$ & $\begin{array}{l}N_{m}(x, y, z)=1+\frac{\left(x-x_{m}\right)\left(y-y_{m}\right)\left(z-z_{m}\right)}{L^{3}} \\
N_{m, y}(x, y, z)=\frac{\left(x-x_{m}\right)\left(z-z_{m}\right)}{L^{3}}\end{array}$ & $\begin{array}{l}N_{m, x}(x, y, z)=\frac{\left(y-y_{m}\right)\left(z-z_{m}\right)}{L^{3}} \\
N_{m, z}(x, y, z)=\frac{\left(x-x_{m}\right)\left(y-y_{m}\right)}{L^{3}}\end{array}$ \\
\hline$n$ & $\begin{array}{l}N_{n}(x, y, z)=1-\frac{\left(x-x_{n}\right)\left(y-y_{n}\right)\left(z-z_{n}\right)}{L^{3}} \\
N_{n, y}(x, y, z)=-\frac{\left(x-x_{n}\right)\left(z-z_{n}\right)}{L^{3}}\end{array}$ & $\begin{array}{l}N_{n, x}(x, y, z)=-\frac{\left(y-y_{n}\right)\left(z-z_{n}\right)}{L^{3}} \\
N_{n, z}(x, y, z)=-\frac{\left(x-x_{n}\right)\left(y-y_{n}\right)}{L^{3}}\end{array}$ \\
\hline$o$ & $\begin{array}{l}N_{o}(x, y, z)=1+\frac{\left(x-x_{o}\right)\left(y-y_{o}\right)\left(z-z_{o}\right)}{L^{3}} \\
N_{o, y}(x, y, z)=\frac{\left(x-x_{0}\right)\left(z-z_{0}\right)}{L^{3}}\end{array}$ & $\begin{array}{l}N_{o, x}(x, y, z)=\frac{\left(y-y_{o}\right)\left(z-z_{0}\right)}{L^{3}} \\
N_{o, z}(x, y, z)=\frac{\left(x-x_{0}\right)\left(y-y_{0}\right)}{L^{3}}\end{array}$ \\
\hline$p$ & $\begin{array}{l}N_{p}(x, y, z)=1-\frac{\left(x-x_{p}\right)\left(y-y_{p}\right)\left(z-z_{p}\right)}{L^{3}} \\
N_{p, y}(x, y, z)=-\frac{\left(x-x_{p}\right)\left(z-z_{p}\right)}{L^{3}}\end{array}$ & $\begin{array}{l}N_{p, x}(x, y, z)=-\frac{\left(y-y_{p}\right)\left(z-z_{p}\right)}{L^{3}} \\
N_{p, z}(x, y, z)=-\frac{\left(x-x_{p}\right)\left(y-y_{p}\right)}{L^{3}}\end{array}$ \\
\hline
\end{tabular}
using (A.10) or (A.6), but by

\section{Table B1}

Shape functions and their derivatives at different nodes of the 8-noded 3D FEM element.

Note: $L$ is the length of the cubic element.

$$
\begin{aligned}
\left(\begin{array}{l}
\hat{u}_{12 x} \\
\hat{u}_{12 y} \\
\hat{u}_{12 z}
\end{array}\right)= & \left(\begin{array}{ccc}
x_{2}-x_{1} & 0 & 0 \\
0 & y_{2}-y_{1} & 0 \\
0 & 0 & z_{2}-z_{1}
\end{array}\right)\left(\begin{array}{l}
\varepsilon_{x x} \\
\varepsilon_{y y} \\
\varepsilon_{z z}
\end{array}\right) \\
& +\left(\begin{array}{ccc}
0 & z_{2}-z_{1} & y_{2}-y_{1} \\
z_{2}-z_{1} & 0 & x_{2}-x_{1} \\
y_{2}-y_{1} & x_{2}-x_{1} & 0
\end{array}\right)\left(\begin{array}{l}
\varepsilon_{y z} \\
\varepsilon_{z x} \\
\varepsilon_{x y}
\end{array}\right) \\
= & \left(\begin{array}{ccc}
\varepsilon_{x x} & \varepsilon_{x y} & \varepsilon_{z x} \\
\varepsilon_{x y} & \varepsilon_{y y} & \varepsilon_{y z} \\
\varepsilon_{z x} & \varepsilon_{y z} & \varepsilon_{z z}
\end{array}\right)\left(\begin{array}{l}
x_{2}-x_{1} \\
y_{2}-y_{1} \\
z_{2}-z_{1}
\end{array}\right)
\end{aligned}
$$

Writing (A.11) in the vector form, we obtain

$\hat{\mathbf{u}}_{\mathrm{ij}}=[\boldsymbol{\varepsilon}] \cdot \mathbf{n} l$

Finally, the relative shear displacement vector (the vector form of (A.9)) can be written as

$\hat{\mathbf{u}}_{i j}^{S}=[\boldsymbol{\varepsilon}] \cdot \mathbf{n} l-(([\boldsymbol{\varepsilon}] \cdot \mathbf{n} l) \cdot \mathbf{n}) \mathbf{n}$

which is Eq. (7) used the text.

\section{Appendix B}

Shape functions used in m-DLSM (see Table B1).

\section{References}

[1] Place D, Mora P. Numerical simulation of localization in a fault zone. Pure Appl Geophys 2000;157:1821-45.

[2] Hazzard JF, Young RP. Simulating acoustic emissions in bonded-particle models of rock. Int J Rock Mech Min Sci 2000;37(5):867-72.

[3] Wang Y, Abe S, Latham S, Mora P. Implementation of particle-scale rotation in the 3-D lattice solid model. Pure Appl Geophys 2006;163(9):1769-85.

[4] Darve F, Nicot F. On incremental non-linearity in granular media: phenomenological and multi-scale views (Part I). Int J Numer Anal Methods Geomech 2005;29:1387-409.

[5] Zhao GF, Fang J, Zhao J. A 3D distinct lattice spring model for elasticity and dynamic failure. Int J Numer Anal Methods Geomech 2011;35:859-85.

[6] Abe S, Place D, Mora P. A parallel implementation of the lattice solid model for the simulation of rock mechanics and earthquake dynamics. Pure Appl Geophys 2004;161(11-12):2265-77.

[7] Zhao GF. Development of micro-macro continuum-discontinuum coupled numerical method. PhD Thesis. EPFL, Switzerland; 2010.

[8] Belytschko T, Loehnert S, Song JH. Multiscale aggregating discontinuities: a method for circumventing loss of material stability. Int J Numer Methods Eng 2007;73(6):869-94.

[9] Kilburn CRJ. Multiscale fracturing as a key to forecasting volcanic eruptions. J Volcanol Geoth Res 2003;125:271-89.

[10] de Borst R. Challenges in computational materials science: multiple scales, multi-physics and evolving discontinuities. Comput Mater Sci 2008;43(1):1-15.

[11] Mullins M, Dokainish MA. Simulation of the (001) plane crack in alpha-Iron employing a new boundary scheme. Philos Mag A 1982;46:771-87.

[12] Hasnaoui A, Van Swygenhoven H, Derlet PM. Dimples on nanocrystalline fracture surfaces as evidence for shear plane formation. Science 2003;300:1550-2.

[13] Tadmor EB, Ortiz M, Phillips R. Quasicontinuum analysis of defects in solids. Philos Mag A 1996;173:1529-63.

[14] Fan J. Multiscale analysis of deformation and failure of materials. New York: Wiley; 2010.

[15] Rabczuk T, Xiao SP, Sauer M. Coupling of mesh-free methods with finite elements: basic concepts and tests results. Commun Numer Methods Eng 2006;22(10):1031-65.

[16] Onate E, Rojek J. Combination of discrete element and finite element methods for dynamic analysis of geomechanics problems. Comput Methods Appl Mech Eng 2004;193:3087-128.

[17] Yan B, Regueiro RA, Sture S. Three-dimensional ellipsoidal discrete element modeling of granular materials and its coupling with finite element facets. Eng Computation 2010;27(4):519-50.

[18] Elmekati A, Shamy UE. A practical co-simulation approach for multiscale analysis of geotechnical systems. Comput Geotech 2010;37(4):494-503.

[19] Rousseau J, Frangin E, Marin P, Daudeville L. Multidomain finite and discrete elements method for impact analysis of a concrete structure. Eng Struct 2009;31(11):2735-43.

[20] Lei Z, Zang M. An approach to combining 3D discrete and finite element methods based on penalty function method. Comput Mech 2010;46(4):609-19. 
[21] Morris JP, Rubin MB, Block GI, Bonner MP. Simulations of fracture and fragmentation of geologic materials using combined FEM/DEM analysis. Int J Impact Eng 2006;33:463-73.

[22] Chen SG, Zhao J. A study of UDEC modelling for blast wave propagation in jointed rock masses. Int J Rock Mech Min Sci 1998;35:93-9.

[23] Munjiza A, Owen DRJ, Bicanic N. A combined finite-discrete element method in transient dynamics of fracturing solids. Eng Comput 1995;12: $145-74$.

[24] Cai M, Kaiser PK, Minami M, Maejima T, Tasaka Y, Kurose H. FLAC/PFC coupled numerical simulation of $\mathrm{AE}$ in large-scale underground excavations. Int J Rock Mech Min Sci 2007;44(4):550-64.

[25] Xiao SP, Belytschko T. A bridging domain method for coupling continua with molecular dynamics. Comput Methods Appl Mech Eng 2004;193: 1645-69.

[26] Shi GH. Manifold method of material analysis. In: Transactions of the 9th army 11 conference on applied mathematics and computing. US Army Research Office, Minneapolis, MN, pp. 57-76.

[27] Otter JRH, Gassell AC, Hobbs RE. Dynamic relaxation (paper no. 6986). In: Proc. inst. civil eng, vol. $35 ; 1966$. p. 633-56.
28] Shi GH. Discontinuous deformation analysis - a new numerical model for the static and dynamics of block systems. Ph.D Thesis. Civil Engineering. Berkeley, University of California; 1988.

[29] Kurumatani M, Terada K. Finite cover method with mortar elements for elastoplasticity problems. Comput Mech 2005;36:45-61.

[30] He L, Ma GW. Development of 3D numerical manifold method. Int J Comput Meth 2010;7(1):107-29.

[31] Ma GW, Hao H, Zhou YX. Modeling of wave propagation induced by underground explosion. Comput Geotech 1988;22(3-4):283-303.

[32] Lu Y. Underground blast induced ground shock and its modelling using artificial neural network. Comput Geotech 2005;32:164-78.

[33] Fan SC, Jiao YY, Zhao J. On modeling of incident boundary for wave propagation in jointed rock masses using discrete element method. Comput Geotech 2004;31:57-66.

[34] Zhou Y, Zhao J, Chong K, Seah CC. Dynamic response and tunnel damage from explosion loading. In: Proc. international symposium on defence construction, Singapore; 2002.

[35] Zhou YX, Arnfinn J. Internal separation distances for underground explosives storage in hard rock. Tunnel Under Space Tech 2009;24:119-25. 